Undine Lübeck, Christian Markurt, Johannes Kochems, Johannes Giehl, Benjamin Grosse, Joachim Müller-Kirchenbauer

\title{
Geschäftsmodelle der Energiewende im regionalen Umfeld
}

Preprint | Submitted manuscript

This version is available at https://doi.org/10.14279/depositonce-11843

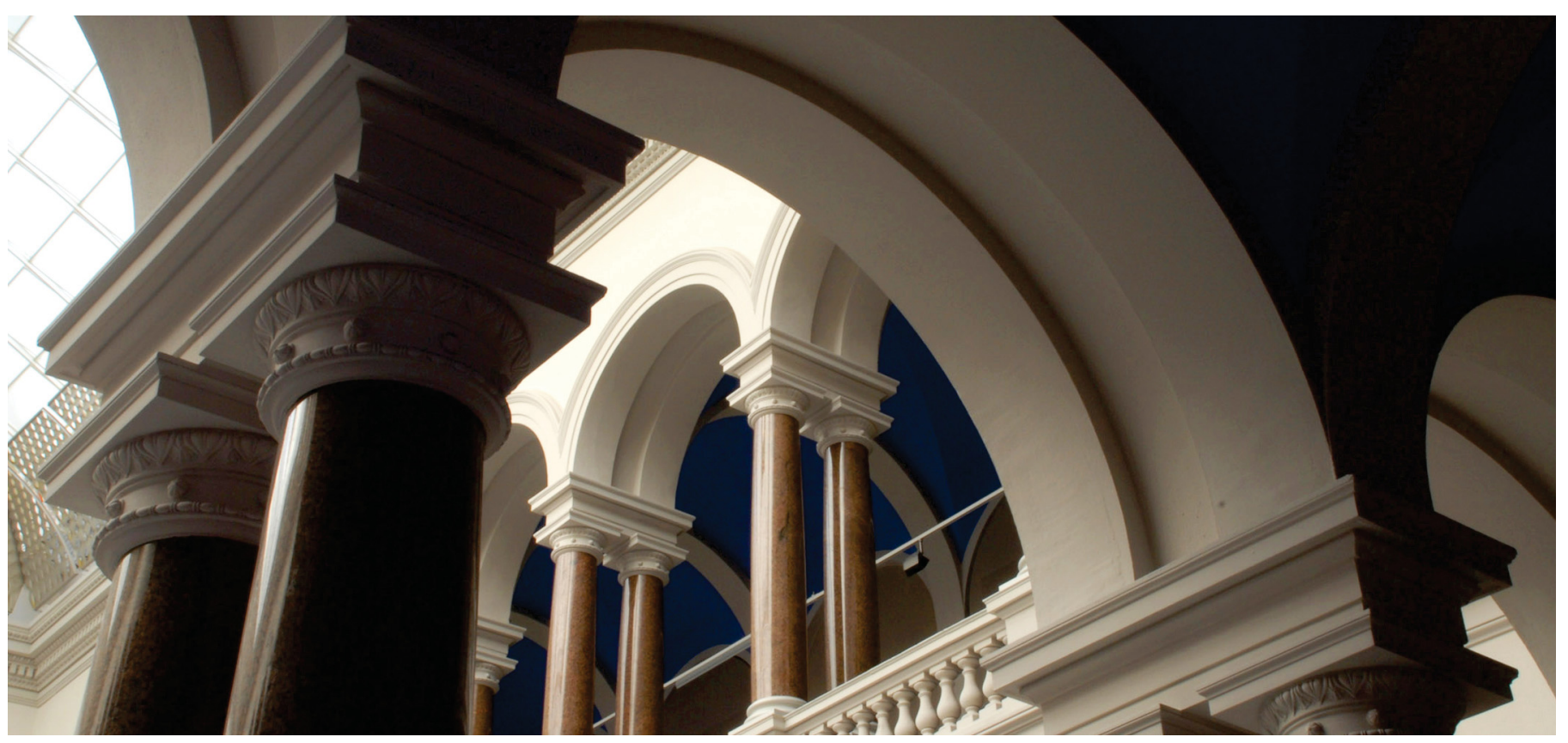

Preprint submitted to Zeitschrift für Energiewirtschaft

Lübeck, Undine; Markurt, Christian; Kochems, Johannes; Giehl, Johannes; Grosse, Benjamin; Müller-Kirchenbauer, Joachim: Geschäftsmodelle der Energiewende im regionalen Umfeld. Preprint at http://dx. doi.org/10.14279/depositonce-11843 (2021). 


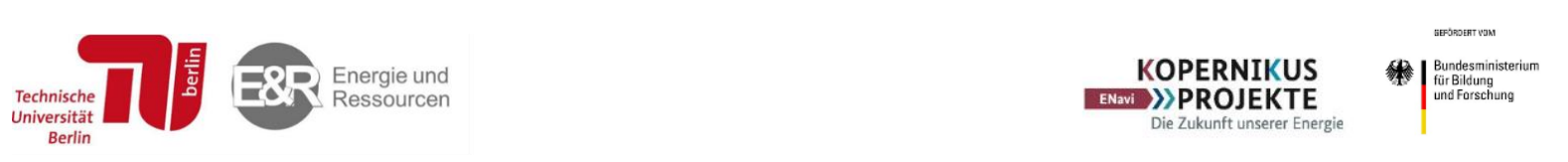

\title{
Geschäftsmodelle der Energiewende im regionalen Umfeld
}

\author{
U. Lübeck, C. Markurt, J. Kochems, J. Giehl, B. Grosse, J. Müller-Kirchenbauer \\ Fachgebiet Energie- und Ressourcenmanagement, Technische Universität Berlin
}

\begin{abstract}
Die Energiewende beeinflusst maßgeblich die Entwicklung energiewirtschaftlicher Wertschöpfung und Geschäftsmodelle. Diese Entwicklung betrifft auch und insbesondere den ländlichen Raum. Vor diesem Hintergrund wird in diesem Papier für die untersuchte "Modellregion Mecklenburg“ eine Bestandsaufnahme der energiewirtschaftlichen Wertschöpfungskette durchgeführt. Ferner wird eine Übertragbarkeit des Geschäftsmodell-Frameworks Energiewirtschaft zur Identifikation von Geschäftsmodellprototypen, d. h. einer Zusammenfassung gleichartiger Geschäftsmodelle, aus Giehl et al. (2020) aufgezeigt. Basierend hierauf werden regionale Wertschöpfungsnetzwerke entwickelt und analysiert, um hieraus in Verbindung mit dem Ansatz der Wertschöpfungscluster nach Porter (1991) Handlungsempfehlungen für die Untersuchungsregion abzuleiten.
\end{abstract}

\begin{abstract}
The energy transformation has a major impact on the development of value creation and business models in the energy industry. This development especially affects rural areas. Therefore, this paper presents a capture of the value chain of energy industry for the examined "model region Mecklenburg". Furthermore, this paper shows the application of the business model framework for the energy transformation (BMFE) from Giehl et al. (2020) to identify prototypical business models, as a summary of similar business models. Based on this identification, regional value creation networks are developed and analyzed. They are used in order to derive recommendations for the model region, in connection with the approach of value creation clusters according to Porter (1991).
\end{abstract}

Key-Words: Geschäftsmodelle, Regionale Wertschöpfung, Wertschöpfungsnetzwerke, Energiewende, Fallstudie, Mecklenburg, business model framework for the energy transformation 


\begin{tabular}{c}
$\begin{array}{c}\text { Technische } \\
\text { Universitat } \\
\text { Berlin }\end{array}$ \\
\hline
\end{tabular}

\section{Inhalt}

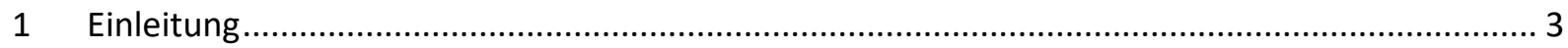

2 Geschäftsmodelle der Energiewirtschaft und regionale Wertschöpfung ..................................... 3

2.1 Geschäftsmodelle der Energiewirtschaft ............................................................................. 4

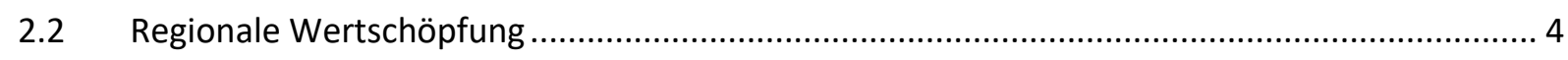

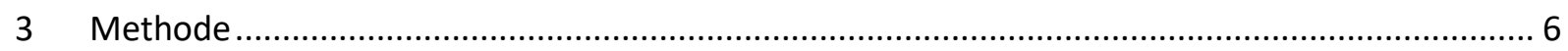

3.1 Bestandsaufnahme der energiewirtschaftlichen Wertschöpfungskette ................................. 6

3.2 Zuordnung von Tätigkeitsbereichen der Unternehmen zu Geschäftsmodellprototypen nach BMFE 8

3.3 Methode zur Bildung von regionalen klassenbasierten Wertschöfungsnetzwerken ................ 9

3.4 Methode zur Bildung von regionalen und unternehmensspezifischen prototypenbasieren

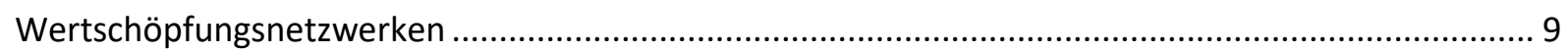

3.5 Entwicklung von Handlungsempfehlungen auf der Basis von Wertschöpfungsclustern.......... 10

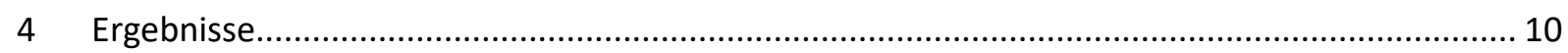

4.1 Bestandsaufnahme der energiewirtschaftlichen Wertschöpfungskette ................................ 10

4.2 Identifizierte Geschäftsmodellprototypen in der Untersuchungsregion .................................. 12

4.3 Bildung eines regionalen klassenbasierten Wertschöpfungsnetzwerks ................................. 13

4.4 Entwicklung von regionalen prototypenbasierten und unternehmensspezifischen

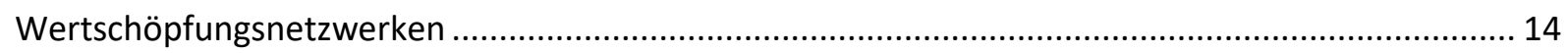

4.5 Ableitung von Handlungsoptionen für das Fallbeispiel...................................................... 17

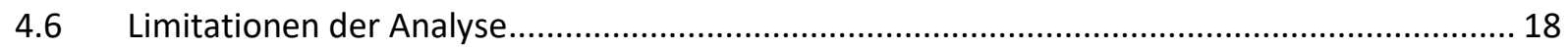

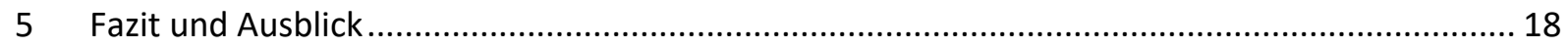

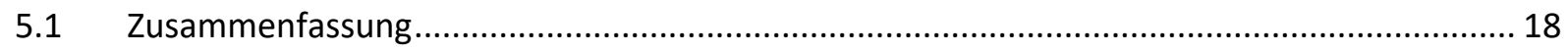

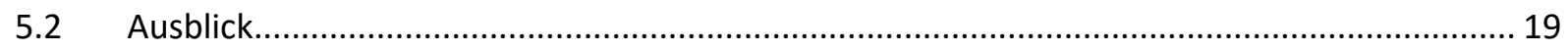

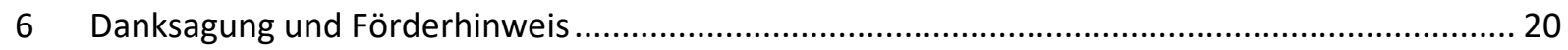

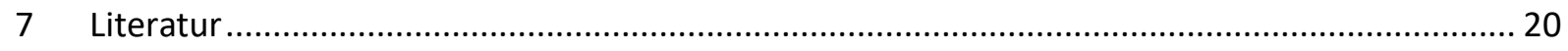

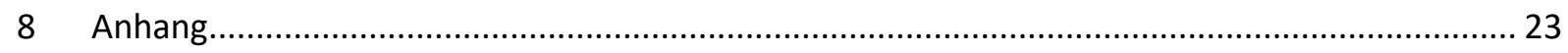

8.1 Anhang 1: Geschäftsmodellklassifizierung der Modellregion Mecklenburg............................. 23

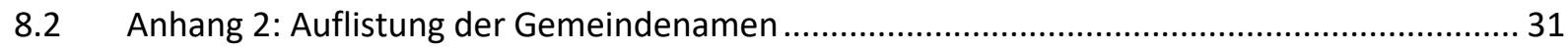




\section{Einleitung}

Die Herausforderungen der Energiewende sowie die Trends Digitalisierung, Dekarbonisierung und Dezentralisierung bedingen Veränderungen der energiewirtschaftlichen Unternehmenslandschaft. Sie lassen neue Geschäftsmodelle entstehen und bedrohen etablierte Unternehmen sowie Geschäftsmodelle im Energiesektor. Zudem führen sie zu einem Wandel von der klassischen Wertschöpfungskettenstruktur hin zu komplexeren Wertschöpfungsnetzwerken mit vielfältigen - auch branchenübergreifenden - Verflechtungen. Vor diesem Forschungshintergrund wurde eine ausführliche und aktuelle Vollaufnahme und Klassifikation von Geschäftsmodellen der Energiewende von Giehl et al. (2020) erstellt. Die strukturierte Geschäftsmodellabbildung der Autoren stützt sich auf ein speziell für den Forschungsgegenstand entwickeltes energiewirtschaftliches Geschäftsmodell-Framework, welches die Systematisierung bestehender und neuer Geschäftsmodelle ermöglicht.

Für die Umsetzung der Energiewende ergeben sich wiederum Herausforderungen und Chancen „vor Ort", beispielsweise in Projekten im regionalen Kontext, welche potenziell regionale Wertschöpfungsbeiträge liefern können. In diesem Zusammenhang soll im vorliegenden Papier untersucht werden, ob und wie sich das methodische Klassifikationsschema aus Giehl et al. (2020) auf den kleinräumigen regionalen Kontext übertragen lässt. Hierbei sollen neben der grundsätzlichen Anwendbarkeit des Schemas energiewirtschaftliche Strukturen und Wertschöpfungspotenziale untersucht werden.

Als Untersuchungsregion wird in diesem Papier die sogenannte „Modellregion Mecklenburg“ gewählt, die sich aus ländlich geprägten Gemeinden der Landkreise Mecklenburgische Seenplatte (MSE) und Nordwestmecklenburg (NWM) im Bundesland Mecklenburg-Vorpommern (MV) zusammensetzt. Die Analysen gliedern sich dabei wie folgt. Zunächst wird in Kapitel 2 ein kurzer Umriss der theoretischen Grundlagen zu regionaler Wertschöpfung und Geschäftsmodellen der Energiewirtschaft gegeben. Anschließend wird in Kapitel 3 auf Basis dieser Hintergründe das methodische Vorgehen entwickelt. Mit der Methodik können einerseits regionale energiewirtschaftliche Wertschöpfungspotenziale ermittelt werden. Andererseits wird die Anwendbarkeit des Klassifizierungsschemas für Geschäftsmodelle auf die realen regionalen Unternehmen bzw. deren Tätigkeitsbereiche untersucht. Es wird analysiert, inwiefern der Ansatz von Wertschöpfungsnetzwerken Anwendung finden kann. Hierbei werden besonders auf erforderliche methodische Erweiterungen des Grundsatzes aus Giehl et al. (2020) betrachtet. Sowohl die Ergebnisse als auch weiterführende Fragestellungen werden abschließend in Kapitel 4 zusammenfassend reflektiert. Ferner werden Handlungsempfehlungen herausgestellt und ein Forschungsausblick gegeben.

\section{Geschäftsmodelle der Energiewirtschaft und regionale Wertschöp- fung}

Im Folgenden werden relevante Grundbegriffe und -zusammenhänge bezüglich der regionalen Wertschöpfung sowie der Geschäftsmodelle der Energiewirtschaft definiert und erläutert. Die Untersuchungsregion wird abschließend ebenso wie der Grundansatz der Wertschöpfungscluster nach Porter (1991, S.151) kurz umrissen. 


\subsection{Geschäftsmodelle der Energiewirtschaft}

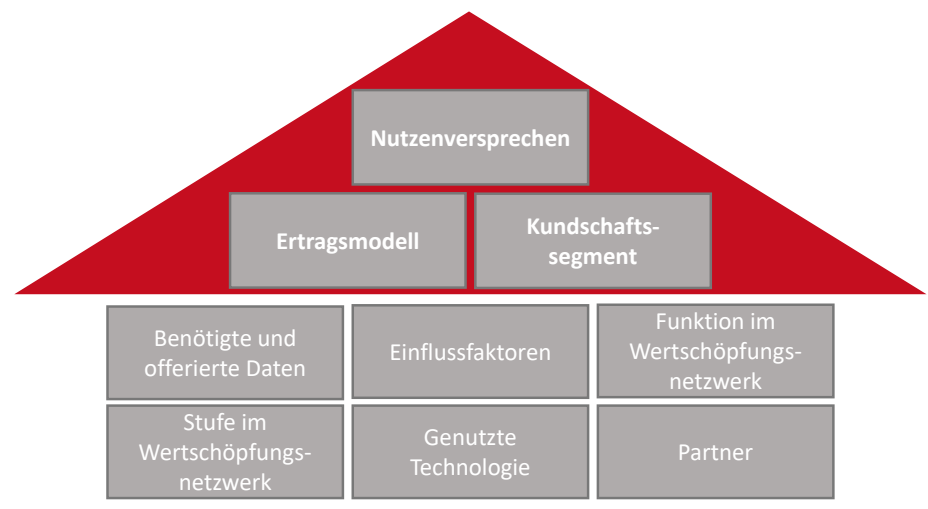

Abbildung 1: Übersicht der Komponenten des business model frameworks for the energy transformation Quelle: Giehl et al. 2020

In dieser Arbeit wird auf das business model framework for the energy transformation (BMFE) der Autoren Giehl et al. (2020) zurückgegriffen. Das BMFE ist angelehnt an vorhandene Frameworks zur Analyse von Geschäftsmodellen - insbesondere den Business Model Canvas (BMC) von Osterwalder und Pigneur (2010). Durch die Synthese weiterer Systematisierungen wie derjenigen von Demil und Lecocq (2010), David Teece (2010) oder Bieger und Reinhold (2011) ist es um weitere Aspekte ergänzt und auf die Energiewirtschaft zugeschnitten worden (Giehl et al., 2020). Damit dient es der gezielten Erfassung und Systematisierung, der in der Energiewirtschaft existierenden Geschäftstätigkeiten und deren Besonderheiten. Es ist in Giehl et al. (2020) erstmals veröffentlicht worden. Das BMFE umfasst dabei die grundlegenden generischen Komponenten Nutzenversprechen, Kundensegment und Ertragsmodell. Diese drei Komponenten sind Bestandteile der meisten Geschäftsmodel-Frameworks. Sie geben eine grundlegende Aussage über die wesentlichen Kernelemente eines Geschäftsmodells bzw. eines Geschäftsmodellprototyps, d. h. einer Zusammenfassung gleichartiger Geschäftsmodelle. Ergänzend dazu enthält das BMFE sechs weitere Komponenten (Abbildung 1) (Giehl et al., 2020).

Insgesamt wurden mit Hilfe dieses Analyseinstruments in einer Vollaufnahme der existierenden energiewirtschaftlichen Geschäftsmodelle durch die Autoren 69 Geschäftsmodellprototypen als Zusammenfassung gleichartiger Geschäftsmodelle beschrieben und wiederum in 17 Geschäftsmodellklassen zusammengefasst (Giehl et al., 2020). Ferner wurden energiewirtschaftliche Wertschöpfungsnetzwerke als Analyseinstrument zur Beschreibung der Wechselwirkungen zwischen Geschäftsmodellprototypen bzw. Geschäftsmodellklassen eingeführt (Giehl et al., 2020). Diese Systematisierungen bilden die Grundlage für die weiterführenden Analysen dieser Arbeit.

\subsection{Regionale Wertschöpfung}

Vom betriebswirtschaftlichen Blickpunkt ist Wertschöpfung als „Beitrag eines Unternehmens zum Volkseinkommen“ (Gabler Wirtschaftslexikon, 2019) zu verstehen. Der Zuwachs an Wert kann dabei durch den Vergleich von Input und Output bemessen werden (Weber, Kabst \& Baum, 2018), der durch die ökonomischen Aktivitäten mit Einfluss auf den Produktwert entsteht (Dyckhoff, 2003). Der Kunde bewertet dabei anhand der sich einstellenden Marktpreise diese Leistungen (Haller, 1997).

Wird dieser unternehmensinterne Betrachtungsansatz auf einen volkswirtschaftlichen Kontext ausgeweitet, kann Wertschöpfung gesamtheitlich als eine „in einzelnen Wirtschaftsbereichen erbrachte wirtschaftliche Leistung" (Gabler Wirtschaftslexikon, 2019) aufgefasst werden. Die Leistungserstellung aller Unternehmen wird demnach gesamtwirtschaftlich relativiert betrachtet. Im Kontext der volkswirtschaftlichen Gesamtrechnung umfasst die Bruttowertschöpfung den Gesamtwert der erzeugten Produktionsgüter abzüglich des Werts der Vorleistungen und liefert damit ein Maß für die Wertschöpfung im volkswirtschaftlichen Sinne (Destatis, 2019). 
Unternehmen interagieren im Rahmen ihrer wertschöpfenden Tätigkeit mit vor- und nachgelagerten Akteuren. Die Reihenfolge der wertsteigernden Arbeitsschritte wird klassischerweise als Wertschöpfungskette aufgefasst (Porter, 1985; Meffert, 1989; Baur \& Kluge, 2000). Durch neu aufkommende, komplexe und angrenzende Branchen miteinbeziehende Verflechtungen der zur Wertschöpfung beitragenden Akteure sind diese zunehmend in Wertschöpfungsnetzwerken aktiv. Beigetragen zum Aufbrechen starrer Produktionsschritte haben in der Energiewirtschaft u. a. Digitalisierung, Dezentralisierung und Dekarbonisierung (Giehl et al., 2020). Dabei ist der strukturelle Aufbau der Wertschöpfungskette bei identischer Technologie weiterhin vorhanden. Die organisatorischen Ausführungsformen der Wertschöpfung verändern sich jedoch hinsichtlich der Akteursvielfalt und der daraus resultierenden Aktivitäten. Diese verlagern sich von unternehmensinternen zu unternehmensexternen Verknüpfungen (Miroschedji, 2002).

Ein Beispiel ist das Messwesen. Zuvor wurde diese Tätigkeit innerhalb eines integrierten Unternehmens absolviert. Inzwischen wird es von dritten Unternehmen durchgeführt und im Zuge des Einsatzes von Smart Metern und damit verbundenen großen Datenmengen bietet es sich an, die Auswertungen auf weitere spezialisierte externe Dienstleister zu übertragen. Diese Form der Wertschöpfung ist bereits durch Porter und dessen Wertsystem angelegt. Der Wertschöpfungsnetzwerkansatz geht allerdings darüber hinaus und kann als Modell der vielfältigen Austauschbeziehungen und Kooperation zwischen Unternehmen als Analyseinstrument eingesetzt werden (Peppard \& Rylander, 2006; Biem \& Caswell, 2008; Bach \& Krüger, 2003).

Ist der regionale Raum, in dem eine wertschöpfende Betrachtung durchgeführt wird, durch klare räumliche Grenzen eingeschränkt, muss dementsprechend auch die Begrifflichkeit der Wertschöpfung auf den Betrachtungsraum angeglichen werden. Das Bundesministerium für Verkehr, Bau und Stadtentwicklung konkretisiert den regionalen volkswirtschaftlichen Wertschöpfungsbegriff als Wertzuwachs verschiedener Wirtschaftsbereiche innerhalb eines abgegrenzten Gebietes. Operationalisierbar ist dieser Zuwachs durch den monetär bewerteten örtlichen Verbleib von Überschüssen, die durch unternehmerische Tätigkeit, Beschäftigte, Geldgeber oder den Staat in dem abgegrenzten Gebiet erwirtschaftet werden (BMVBS, 2011). Für die weiteren Untersuchungen dieses Papiers ist es daher vonnöten, eine regionale Begrenzung der Untersuchungsregion zu definieren.

Untersuchungsgebiet der Analyse ist die "Modellregion Mecklenburg". Diese besteht aus sieben Gemeinden im Landkreis NWM sowie zwölf Gemeinden im Landkreis MSE im Bundesland MV. Beide Regionen beteiligen sich am Forschungsprojekt ENavi. Eine Auflistung der untersuchten Gemeinden ist im Anhang $8.2 \mathrm{zu}$ finden. Die Gemeinden sind durch verhältnismäßig strukturschwache ländliche Räume und die demografischen sowie wirtschaftlichen Folgen der Wende in Ostdeutschland sozioökonomisch geprägt (BBSR, 2012). Aufgrund ihrer wirtschaftlichen Struktur sind die Gemeinden gut geeignet für die Frage, wie die Umstellung des Energiesystems zum Gewinn für die dortige Bevölkerung werden und eine Perspektive wirtschaftlicher Entwicklung bieten kann.

Der abgegrenzte Untersuchungsraum kann nun wiederum hinsichtlich seiner wertschöpfenden Aktivitäten gesondert betrachtet werden, um vorhandene energiewirtschaftliche Potentiale zu identifizieren. Methodisch kann hierbei das Konzept des Diamantenmodells nach Porter $(1991$, S.151) angewendet werden, um regionalwirtschaftliche Entwicklungspotenziale zu identifizieren. Das Modell von Porter beschäftigt sich dabei mit der Frage, warum Unternehmen bestimmter Branchen aus definierten Regionen gebündelt wettbewerbsfähiger sind als andere (Porter, 1991). Nach Porter können in lokalen Wertschöpfungssystemen durch effektive Koordination und Bündelung in einem Gebiet Vorteile in Bezug auf Produktivität, Flexibilität und damit Effizienz erreicht werden. Dies geschieht, indem im regionalen Gebiet die wesentlichen Funktionen der Wertschöpfungskette vereint werden (Steinle \& Schiele, 
2010). Porter (1991) sieht die positiven Wechselwirkungen von vier elementaren Bestimmungsfaktoren als maßgelblich für das Entstehen von Wettbewerbsvorteilen durch Branchenanhäufungen und Clusterbildungen:

Strategie, Struktur und Wettbewerb: Die Konkurrenz zwischen Unternehmen ähnlicher Ziele und Strategien steigert Innovations- und Wettbewerbsfähigkeit.

Nachfragebedingungen: Das Vorhandensein von Nachfrage nach Produkten und Dienstleistungen der Branche muss gegeben sein.

Unterstützende Branchen: Die Existenz und Nähe von vor- und nachgelagerten Wirtschaftszweigen fördert produktive Wertschöpfungsstrukturen durch Kooperationen.

Faktorbedingungen: Die Verfügbarkeit von effizienten Produktionsfaktoren besteht als Grundlage der Wertschöpfung und Wettbewerbsfähigkeit.

Die Faktoren stehen dabei in einem dynamischen Zusammenhang der wechselseitigen Beeinflussung, welche sich durch geografische Nähe zusätzlich verstärkt (Porter, 1991). Die Bildung von wettbewerbsfähigen Clustern lässt sich demnach durch die Ausprägung der einzelnen Faktoren sowie deren räumlich verstärkte gegenseitige Beeinflussung erklären (Porter, 1991).

\section{Methode}

Die Zielsetzung dieses Papiers besteht in der Überprüfung der Anwendbarkeit der Geschäftsmodellklassifizierung nach Giehl et al. (2020) für einen abgegrenzten regionalen Raum sowie reale Unternehmen. In diesem Papier kommt der Klassifizierungsansatz innerhalb der ländlichen „Modellregion Mecklenburg“" (s. Kapitel 2.2) zum Einsatz. Hierfür wird das folgende fünfstufiges analytisches Vorgehen angewandt:

1. Den Ausgangspunkt der Analysen bildet eine Erfassung des Status quo der energiewirtschaftlichen Wertschöpfung.

2. Anhand der Tätigkeitsbeschreibung der energiewirtschaftlich tätigen Unternehmen erfolgt anschließend eine Zuordnung von deren Geschäftsmodellen zu Geschäftsmodellprototypen, um die Anwendbarkeit des Klassifikationsschemas auf Giehl et al. (2020) für einen kleinräumigen regionalen Betrachtungsraum zu demonstrieren.

3. Basierend auf dieser Zuordnung wird anschließend ein Wertschöpfungsnetzwerk auf Ebene von Geschäftsmodellklassen für die Untersuchungsregion entwickelt.

4. Im Folgenden wird ein Wertschöpfungsnetzwerk auf Ebene von Geschäftsmodellprototypen für ein konkretes unternehmerisches Beispiel entwickelt, um die Anwendbarkeit auf reale Unternehmen und Geschäftsmodelle zu demonstrieren.

5. An die Analyse knüpfen Untersuchungen hinsichtlich der Entwicklungspotenziale für die energiewirtschaftliche Wertschöpfung der Modellregion an, wobei auf das Konzept der Wertschöpfungscluster nach Porter (1991) zurückgegriffen wird.

\subsection{Bestandsaufnahme der energiewirtschaftlichen Wertschöpfungskette}

Die Erfassung der energiewirtschaftlichen Wertschöpfung in der Modellregion wird unter Zuhilfenahme der Wertschöpfungskette der Energiewirtschaft nach Porter (1991) durchgeführt. Um die Besonderheiten der Energiewirtschaft im ländlichen Raum abzubilden, erfährt die Wertschöpfungskette eine Anpassung. Energiewirtschaftlich tätige Akteure werden durch eine Erhebung von Sekundärdaten erfasst und können einer Stufe zugeordnet werden. Dies erlaubt eine anschließende Beurteilung, wie umfangreich Unternehmen vor Ort im Sinne der adaptierten Wertschöpfungskette vertreten sind. 
Die Wertschöpfungskette der Energiewirtschaft umfasst zunächst die vier Grundstufen Erzeugung, Handel, Transport (und Verteilung) sowie Vertrieb und kann für verschiedene Energieträger und Technologien angewendet werden. Anders als beim Wert(schöpfungs)kettenansatz nach Porter (1991) werden Leistungen über alle Stufen hinweg und für alle relevanten Unternehmen analysiert.

Die Grundstufen der Wertschöpfungskette werden in diesem Papier teilweise weiter aufgegliedert, um den Besonderheiten des ländlichen Raums Rechnung zu tragen. Die "Modellregion Mecklenburg" ist laut der statistischen Klassifikation von BBSR, EUROSTAT und OECD als ländlicher Raum einzustufen. Insofern ergeben sich - auch durch das vorhandene Flächenpotenzial - energiewirtschaftliche Perspektiven insbesondere hinsichtlich des Ausbaus von Anlagen zur Erzeugung von Erneuerbaren Energien (Keppler, Nölting \& Schröder, 2011), welche als Ausgangsbasis für die Entwicklung regionaler Wertschöpfungscluster dienen können. Um dies zu berücksichtigen, wird die Stufe Erzeugung nach den Vorschlägen über den Anlagen-Lebenszyklus der GWS (2016) ausdifferenziert. Unternehmen der Wissensökonomie sind insbesondere in Agglomerationsräumen vorzufinden. Die Stufen Handel und Vertrieb sind demnach hypothetisch weniger stark im ländlichen Raum repräsentiert und werden entsprechend nicht weiter ausdifferenziert. Der Transport wird ebenfalls nicht weiter ausdifferenziert. Klassische Vertriebsaktivitäten werden zusammengefasst. Energieberatungen als etabliertes Geschäftsmodell werden als relevante Tätigkeit mitaufgenommen und von klassischen Vertriebsmodellen abgegrenzt. Neue Vertriebsmodelle werden aufgrund ihrer Heterogenität den klassischen zugerechnet. Angrenzende Aktivitäten bzw. Kundenservices werden nicht erfasst, um damit den Analysefokus auf die Energiewirtschaft im engeren Sinne zu legen. Ein Erfolgsfaktor für die Entwicklung von Wertschöpfungsclustern wird in der Ansiedlung Cluster-bezogener Forschung und Entwicklung gesehen (Biem \& Caswell, 2008). Diese Kategorie wird daher gesondert aufgenommen. Abbildung 2 zeigt die angepasste Wertschöpfungskette der Energiewirtschaft für den ländlichen Raum, die Stufen in kursiv sind als Teilmengen zu verstehen.

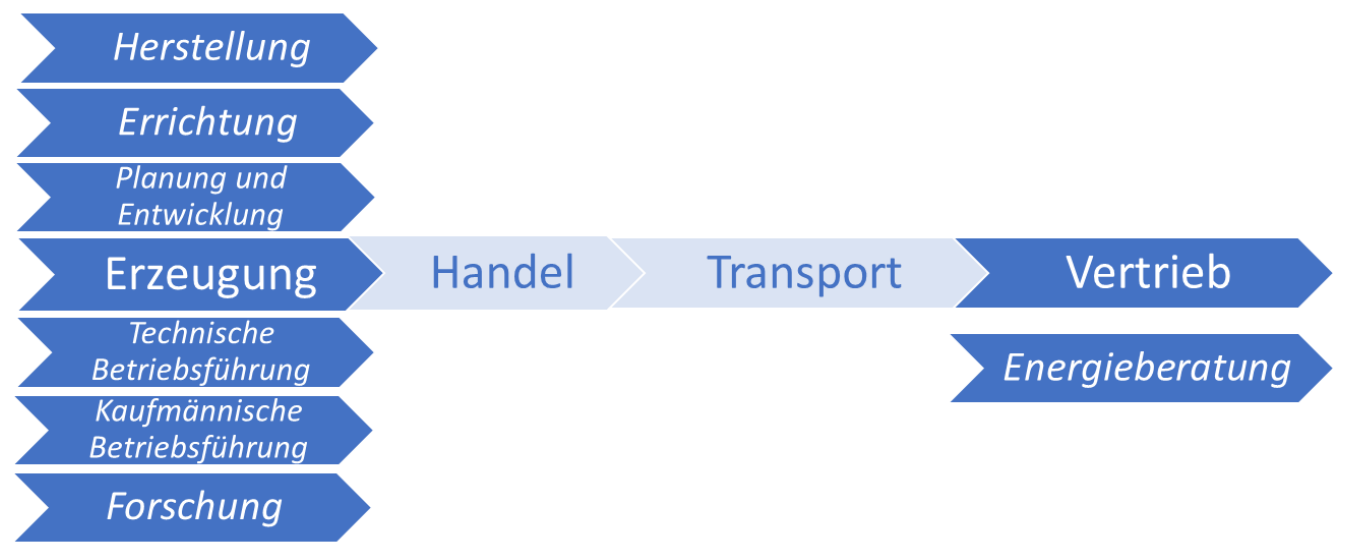

Abbildung 2: Die angepasste Wertschöpfungskette der Energiewirtschaft für den ländlichen Raum Quelle: Eigene Darstellung

Die Wertschöpfung wird anhand zweier Indikatoren operationalisiert:

- Zum einen wird Wertschöpfung anhand des Unternehmenssitzes der energiewirtschaftlich tätigen und regional ansässigen Unternehmen bemessen, da die Unternehmen durch Gewinne regionale Steuerabgaben und Beschäftigte induzieren.

- Zum anderen werden regional vorhandene erneuerbare Erzeugungsanlagen als Wertschöpfungsindikatoren aufgefasst, da sie, auch wenn die Betreiber nicht vor Ort ansässig sind, vor allem durch technische Dienstleistungen einen regionalen Mehrwert erzeugen.

Als zentrale Datenquellen für das Vorhandensein von Unternehmen werden das Handelsregister und Webpräsenzen der Unternehmen herangezogen. Das Handelsregister (2019) verzeichnet angemeldete 
Kaufleute und Gesellschaften. Das Handelsregister wird nach Energie-bezogenen Unternehmensnamen durchsucht und die Webseiten der identifizierten Unternehmen im Internet auf Leistungsangebote im Energiebereich hin untersucht. Da nicht-selbstständige Betriebsteile nicht im Handelsregister erscheinen, wird unter Verwendung einschlägiger Suchbegriffe flankierend im Internet nach lokalen Unternehmen gesucht. Die Schlagworte der Suche entsprechen dem Untersuchungsgegenstand der Studie und umfassen die Begriffe "Energie", "Strom", "Gas", „Wärme“. Davon ausgehend werden weitere äquivalente und verwandte Begriffe abgeleitet und mithilfe einer Schlagwortmatrix zu geeigneten Such-Strings kombiniert. Erzeugungsanlagen wurden mittels der Geodatenportale der Landkreise NWM (Landkreis Nordwestmecklenburg, 2019) und MSE (Landkreis Mecklenburgische Seenplatte, 2019), Informationen des Ministeriums für Landwirtschaft und Umwelt MV über Biomasseanlagen (Ministerium für Landwirtschaft und Umwelt, 2019) sowie eine Anfrage im Landtag MV über Windkraftanlagen (Landtag Mecklenburg-Vorpommern, 2018) und die Anlagenstammdaten des Übertragungsnetzbetreibers $50 \mathrm{Hertz}$ (Netztransparenz.de, 2018) identifiziert.

\subsection{Zuordnung von Tätigkeitsbereichen der Unternehmen zu Geschäftsmodellproto- typen nach BMFE}

Um den regionalen Unternehmen einen Geschäftsmodellprototypen zuzuordnen, kommt das Framework von Giehl et al. (2020) zum Einsatz. Hierzu wird jedes regionale Unternehmen hinsichtlich der Ausprägungen relevanter Komponenten des BMFE individuell untersucht. Es werden die drei Hauptkomponenten Nutzenversprechen, Ertragsmodell und Kundensegment analysiert, durch die sich nach Giehl et al. (2020) ein Geschäftsmodellprototyp eindeutig beschreiben lässt. Von den weiteren Komponenten werden nur drei der sechs von Giehl et al. (2020) definierten genauer untersucht. Die regionalen Unternehmen werden hinsichtlich der genutzten Technologie, der Stufe im Wertschöpfungsnetzwerk und ihren (Geschäfts-)Partnern analysiert. ${ }^{1}$ Insbesondere die genutzte Technologie sowie die Stufe im Wertschöpfungsnetzwerk schärfen die Abgrenzung gegenüber anderen Geschäftsmodellprototypen. Das Geschäftsmodellelement Partner gewinnt vor allem Bedeutung für den Aufbau von Wertschöpfungsnetzwerken auf Ebene von Geschäftsmodellprototypen (s. Kapitel 3.4), da hierdurch die Verflechtungen zu anderen Geschäftsmodellprototypen bzw. den dahinterstehenden Unternehmen aufgezeigt werden. Sofern für eine der erhobenen Komponente keine Zuordnung zu einer der vorhandenen Komponentenausprägungen aus der Menge möglicher Ausprägungen aus Giehl et al. (2019) möglich ist, wird eine neue Ausprägung für die jeweilige Komponente definiert.

\footnotetext{
${ }^{1}$ Die drei Komponenten Einflussfaktoren, Funktion im Wertschöpfungsnetzwerk und benötigte Daten werden nicht erfasst. Einflussfaktoren sind nach Giehl et al. auf nationaler Ebene angelegt. Die Funktion im Wertschöpfungsnetzwerk und die benötigten Daten bedürfen zudem einer individuellen Analyse von jedem Unternehmen in der Untersuchungsregion. Aus diesem Grund wird auf die generischen Ausprägungen dieser Komponenten nach Giehl et al. (2019 und 2020) verwiesen.
} 


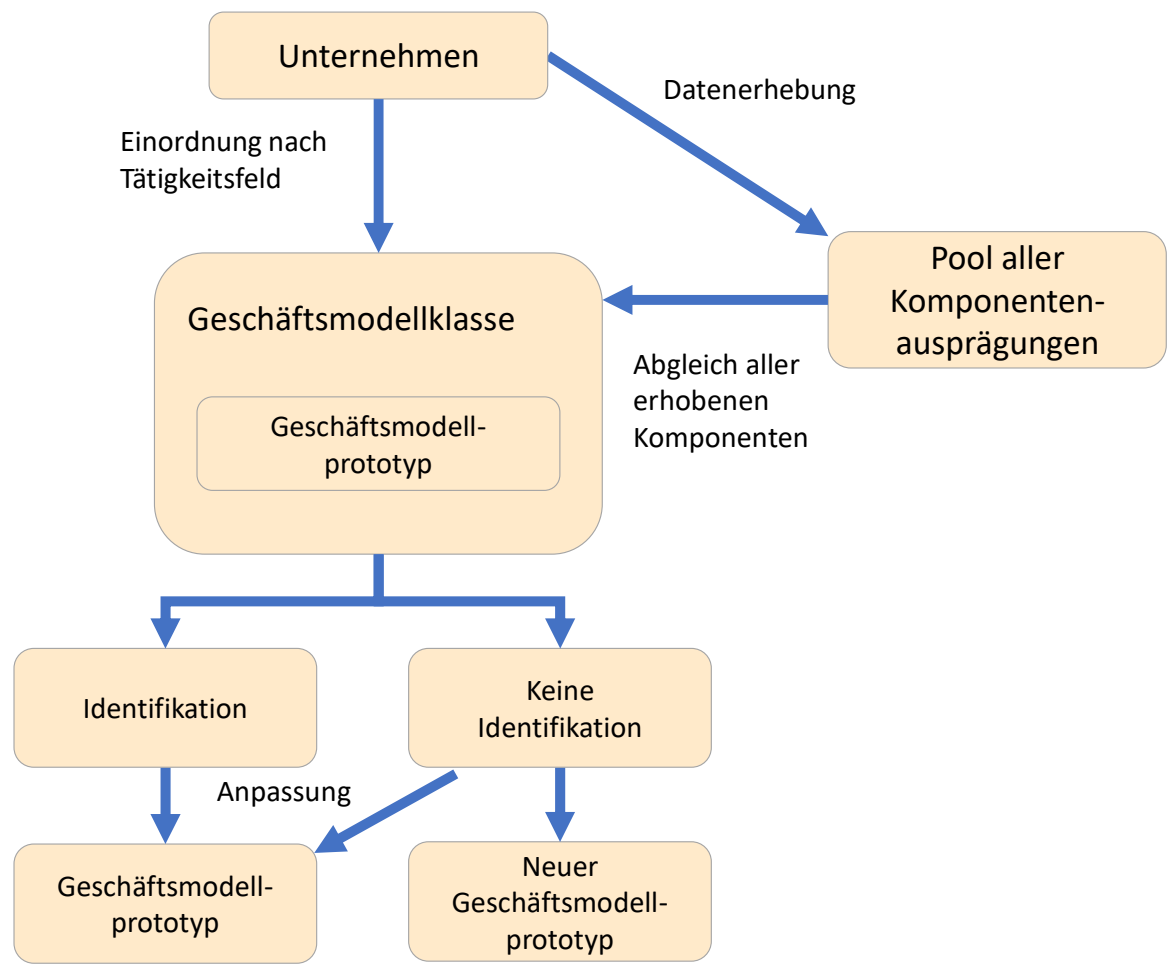

Abbildung 3: $\quad$ Vorgehen bei der Zuordnung zu Geschäftsmodellprototypen Quelle: Eigene Darstellung

Die sich ergebenden Komponentenausprägungen werden anschließend mit denen der von Giehl et al. (2019) definierten Geschäftsmodellprototypen abgeglichen. Stimmen die Ausprägungen der untersuchten BMFE-Komponenten mit denen eines Geschäftsmodellprototyps überein, so lässt sich das Geschäftsmodell des Unternehmens eindeutig diesem zuordnen und damit implizit auch einer Geschäftsmodellklasse. Findet sich für die erhobenen Komponentenausprägungen kein existierender Geschäftsmodellprototyp, so wird ein neuer definiert.

\subsection{Methode zur Bildung von regionalen klassenbasierten Wertschöfungsnetzwerken} Durch eine Zuordnung der Tätigkeitsbereiche der Unternehmen zu Geschäftsmodellprototypen ist implizit auch eine Zuordnung zu einer der insgesamt 17 Geschäftsmodellklassen gegeben. Zur Systematisierung der Geschäftsmodellklassen innerhalb und außerhalb der Untersuchungsregion wird ein klassenbasiertes Wertschöpfungsnetzwerk basierend auf Giehl et al. (2020) entwickelt. In dieses Wertschöpfungsnetzwerk werden weitere relevante Informationen integriert. Dies umfasst einerseits eine Differenzierung von innerhalb und außerhalb der Untersuchungsregion vorhandenen Geschäftsmodellklassen sowie andererseits die Information zur Anzahl der je Geschäftsmodellklasse vertretenen Geschäftsmodellprototyen.

Das Wertschöpfungsnetzwerk dient somit als Analyseinstrument, aus dem sich Aussagen hinsichtlich des regionalen energiewirtschaftlichen Entwicklungspotenzials untersuchen und veranschaulichen lassen. Hieraus können Handlungsempfehlungen zur potenziellen Generierung zusätzlicher Wertschöpfungsbeiträge abgeleitet werden (Giehl et al., 2020). Dafür wird auch der Ansatz von Wertschöpfungsclustern nach Porter (1991) herangezogen.

\subsection{Methode zur Bildung von regionalen und unternehmensspezifischen prototypen- basieren Wertschöpfungsnetzwerken}

Zur Untersuchung der Zusammenhänge zwischen Geschäftsmodelprototypen der untersuchten Unternehmen und zur Demonstration der Anwendbarkeit auf reale Unternehmen wird für einen in der Modellregion häufig anzutreffenden Geschäftsmodellprototypen ein Wertschöpfungsnetzwerk auf Ebene 
von Geschäftsmodellprototypen entwickelt. Die Grundlage für die Verknüpfungen zwischen den Prototypen liefern die Komponenten Partner, Kunden, Funktion im Wertschöpfungsnetzwerk und benötigte Daten. Die Ausprägungen dieser Komponenten werden ausschließlich für den repräsentativen Geschäftsmodellprototyp der Modellregion und durch das Heranziehen der allgemeinen Ausprägungen nach Giehl et al. (2019) erhoben. Auf Basis der vier Komponenten wird ein Wertschöpfungsnetzwerk entworfen, welches sich um den ausgewählten Prototyp aufspannt und seine Positionierung zu anderen regional auftretenden Geschäftsmodellen widerspiegelt.

Das Wertschöpfungsnetzwerk wird zunächst auf der Grundlage der Systematik aus Giehl et al. (2020) aufgebaut. Anschließend wird die Anwendbarkeit des prototypbasierten Wertschöpfungsnetzwerks durch eine konkrete Fallstudie überprüft. Hierfür wird ein Beispielunternehmen aus der Modellregion ausgewählt und ein Wertschöpfungsnetzwerk aufgebaut, das realwirtschaftliche Verflechtungen abbildet. Mittels Triangulation erfolgt so einen Vergleich unterschiedlicher qualitativer methodischer Lösungsansätze, welcher als Gütekriterium der Forschung aufgefasst werden kann (Mayring, 2016).

Das für die Fallstudie ausgewählte Unternehmen wird gezielt einer Datenerhebung bezüglich Kooperationen, wie Partnern, Kunden, Lieferanten etc., im Rahmen eines Interviews unterzogen. Dabei werden grundsätzlich nur die direkten Netzwerkpartner des Tätigkeitsbereichs des Unternehmens bzw. Geschäftsmodellprototyps erhoben. Eine Erweiterbarkeit um indirekt verbundene Geschäftsmodellprototypen ist aber möglich. Auf Wunsch des in der Fallstudie betrachteten Unternehmens hin werden Unternehmensnamen anonymisiert.

\subsection{Entwicklung von Handlungsempfehlungen auf der Basis von Wertschöpfungsclus- tern}

Auf Grundlage des in Kapitel 2.2 eingeführten Diamantenmodells nach Porter (1991) können durch die vier Wettbewerbsfaktoren Handlungsempfehlungen für ein potentielles regionales Wertschöpfungscluster gegeben werden. Hierfür werden die gewonnenen Erkenntnisse aus den Wertschöpfungsnetzwerken dazu genutzt, die Strategie, die Struktur und den Wettbewerb der regionalen Unternehmenslandschaft zu bewerten und diese auf Vollständigkeit und Lücken zu überprüfen. Folglich lassen sich Potentiale und Defizite hinsichtlich der vorhandenen Geschäftsmodelle auffinden sowie Aussagen ableiten, wie durch unterstützende nach- und/oder vorgelagerte Branchen Wertschöpfungsbeiträge des regionalen Wertschöpfungsnetzwerks ausgeweitet werden könnten. Gleichermaßen eröffnet die Geschäftsmodellklassifikation und der Aufbau der Wertschöpfungsnetzwerke auch ein Bild von potentiellem Vorhandensein regionaler Faktorbedingungen. Wenn beispielsweise ein Geschäftsmodell vermehrt in der Region identifiziert wird, lässt das nach Porter auf eine hohe Wettbewerbsintensität zwischen Unternehmen des gleichen Geschäftsmodells und das Vorhandensein bestimmter Ressourcen und Produktionsfaktoren sowie der Nachfrage danach schließen.

\section{Ergebnisse}

Im Folgenden werden zunächst die Ergebnisse der ausführlichen Bestandsaufnahme der energiewirtschaftlichen Wertschöpfungskette für die Untersuchungsregion „Modellregion Mecklenburg“ dargelegt. Es schließen sich Ausführungen dazu an, welche Geschäftsmodellprototypen in der Untersuchungsregion identifiziert werden. Nachfolgend werden regionale Wertschöpfungsnetzwerke auf unterschiedlichen Analyseebenen entwickelt und Handlungsoptionen für regionale Wertschöpfungspotenziale abgeleitet. Eine kritische Würdigung der verwendeten methodischen Ansätze sowie der erzielten Ergebnisse schließt das Kapitel ab.

\subsection{Bestandsaufnahme der energiewirtschaftlichen Wertschöpfungskette}

Diejenigen Unternehmen, die im Rahmen der Bestandsaufnahme identifiziert wurden, werden gemäß der in Kapitel 3.1 dargelegten Vorgehensweise, basierend auf ihrer relevanten Tätigkeit den jeweiligen 
Stufen der (ausdifferenzierten) energiewirtschaftlichen Wertschöpfungskette zugeordnet. Hierbei sind mehrfache Wertaktivitäten möglich. Ferner werden Wertschöpfungsbeiträge aus Erzeugungsanlagen ermittelt, welche nachfolgend zunächst dargestellt sind.

Für den Landkreis NWM kann Wertschöpfung aus der Energieerzeugung mittels Windenergie und Biogas mit einem relativen Schwergewicht auf der Windenergie festgestellt werden. Die Wertschöpfungsstufe der Erzeugung ist somit regional vorhanden. Auch für den Landkreis MSE kann regionale Wertschöpfung in den Bereichen Biogas und Windenergie konstatiert werden. Die installierten Leistungen zur Biogasstromerzeugung sind weit größer als in NWM. Der lokale Windpark Bütow/Zepkow führt ebenso zu größeren Windenergiebeiträgen. Photovoltaik-Freiflächenanlagen finden sich in keiner der beiden Teilregionen.

Die Anzahl der jeweils gefundenen Wertaktivitäten der erfassten Unternehmen ist in Tabelle 1 dargestellt. Aus der Tabelle wird ersichtlich, dass auf fast jeder Wertschöpfungsstufe örtliche Unternehmen relevante Tätigkeiten ausüben. Die Grundstufe Handel ist nicht repräsentiert. Die Aktivität der Energieberatung ist lediglich durch ein Unternehmen repräsentiert.

In den Umlandgemeinden Schwerins in NWM gibt es absolut mehr Wertaktivitäten als in der Region MSE. NWM besitzt herstellende Unternehmen, die potenziell Ausgangspunkt für integrierte Wertschöpfungsnetzwerke sein könnten. Wenngleich es mehrere herstellende Betriebe gibt, so fehlt der Zusammenhang einer produktbezogenen Wertschöpfungskette. Die Herstellung in NWM ist jedoch fragmentiert und zentriert sich nicht um eine Erzeugungsform; alle Produkte gehen an unterschiedliche Leistungsnehmer. In der Region MSE steht die Vielzahl an Erzeugungsanlagen im Kontrast zur geringeren Menge örtlich ansässiger Unternehmen. Es gibt zwar mehrere Unternehmen, welche die Erzeugungsanlagen kaufmännisch betreiben, aber nahezu kein Unternehmen bietet dazugehörige Dienstleistungen an. Entsprechend der Datenerhebung gibt es in NWM 33 relevante Tätigkeiten und in der MSE 29.

Tabelle 1: Anzahl der relevanten Aktivitäten in den Untersuchungsgemeinden

\begin{tabular}{c|cccccccc} 
& Herstellung Errichtung & $\begin{array}{c}\text { Planung \& } \\
\text { Entwicklung }\end{array}$ & $\begin{array}{c}\text { Techn. } \\
\text { Betriebs- } \\
\text { führung }\end{array}$ & $\begin{array}{c}\text { Kaufm. } \\
\text { Betriebs- } \\
\text { führung }\end{array}$ & Forschung & Handel & $\begin{array}{c}\text { Vertrieb (+ } \\
\text { Energiebera- } \\
\text { tung) }\end{array}$ \\
\hline $\begin{array}{c}\text { Gemein- } \\
\text { den in } \\
\text { NWM }\end{array}$ & 5 & 9 & 1 & 6 & 3 & 0 & 0 & 2 \\
$\begin{array}{c}\text { Gemein- } \\
\text { den in } \\
\text { MSE }\end{array}$ \\
2
\end{tabular}




\subsection{Identifizierte Geschäftsmodellprototypen in der Untersuchungsregion}

Anknüpfend an die Erfassung von Unternehmen und deren Tätigkeitsbereichen wir nachfolgend eine Zuordnung dieser zu Geschäftsmodellprototypen vorgenommen. Im Ergebnis kann dem überwiegenden Tätigkeitsbereich jedes regionalen Unternehmens genau ein prototypisches Geschäftsmodell aus der Menge von Geschäftsmodellprototypen von Giehl et al. (2020) zugeordnet werden. Somit ist eine trennscharfe Abgrenzung der Zuordnung zu Geschäftsmodellprototypen gegeben und das BMFE hat sich für die Untersuchungsregion als sehr zielführendes Analyseinstrument erwiesen. Es werden keine neuartigen Prototypen festgestellt, was - zumindest für die betrachtete Region - grundsätzlich als Bestätigung der Vollaufnahme aus Giehl et al. (2020) gewertet werden kann.

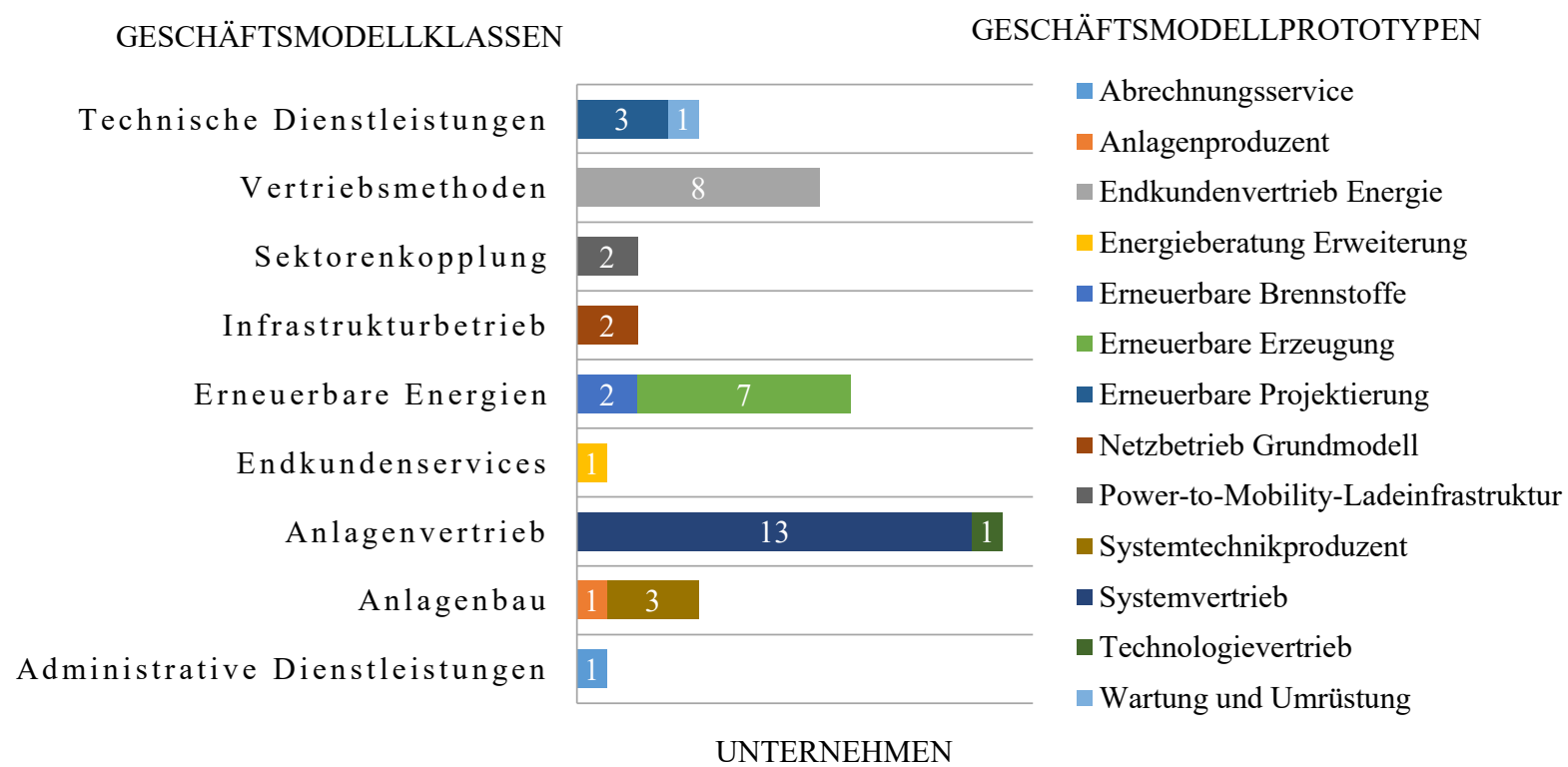

Abbildung 4: Identifizierte Geschäftsmodellprototypen in der Modellregion Mecklenburg Quelle: Eigene Darstellung unter Verwendung von Giehl et al. (2020)

In Abbildung 4 sind die Ergebnisse der Zuordnung der überwiegenden Tätigkeitsbereiche der regionalen Unternehmen zu Geschäftsmodellprototypen dargestellt und die Prototypen ferner nach Giehl et al. (2020) in Geschäftsmodellklassen gruppiert. Am stärksten repräsentiert sind die Geschäftsmodellprototypen Systemvertrieb, Endkundenvertrieb Energie und Erneuerbare Erzeugung sowie deren korrespondierende Geschäftsmodellklassen Anlagenvertrieb, Vertriebsmethoden und Erneuerbare Energien. Der Prototyp Systemvertrieb wird hierbei - wie auch im vorigen Kapitel ersichtlich - überwiegend von Heizungsinstallateuren und Haustechnikvertrieben bedient. Der Prototyp Erneuerbare Erzeugung ist durch Windpark- und Biogasanlagenbetreibergesellschaften repräsentiert. Dem Prototypen Endkundenvertrieb Energie werden hauptsächlich Tankstellen zugeordnet. 
Tabelle 2: Ergänzende und alternative Ausprägungen zu einzelnen Geschäftsmodellprototypen nach Giehl et al. (2020)

\begin{tabular}{|l|l|l|l|l|l|l|}
\hline Geschäftsmodellprototyp & Komponente & $\begin{array}{l}\text { Ergänzende } \\
\text { Ausprägungen }\end{array}$ & Biomasseanlagen \\
\hline Systemvertrieb & Technologie & & Energieholzwerk* \\
\hline Erneuerbare Brennstoffe & Technologie & Kraftstoffbereitstellung \\
\hline Endkundenvertrieb Energie & Nutzenversprechen & Energiedienstleister & \\
\hline Erneuerbare Erzeugung & Partner & Erzeuger & \\
\hline Prüfdienstleistungen & Kundensegment & Erzeuger & \\
\hline $\begin{array}{l}\text { Energieberatung Erweite- } \\
\text { rung }\end{array}$ & Kundensegment & & \\
\hline *Die Ausprägung kann verallgemeinernd anstelle des Pelletwerks aufgeführt werden. & \\
\hline Quelle: Eigene Darstellung unter Verwendung von Giehl et al. (2019 und 2020) & \\
\hline
\end{tabular}

Während für alle Geschäftsmodelle eine Zuordnung zu existierenden Geschäftsmodellprototypen nach Giehl et a. (2020) möglich ist, sind für wenige Komponentenausprägungen Anpassungen zu treffen. Die überwiegende Zahl der Anpassungen kann aus dem Pool vorhandener Komponentenausprägungen erfolgen. Dies umfasst die folgenden Komponenten sowie deren Ausprägungen:

Zu den identifizierten Geschäftsmodellprototypen je Haupttätigkeitsbereich eines Unternehmens findet sich im Anhang 8.1 eine detaillierte Aufstellung. In dieser sind auch die jeweiligen Komponentenausprägungen sowie vorgenommene Anpassungen aufgelistet.

\subsection{Bildung eines regionalen klassenbasierten Wertschöpfungsnetzwerks}

In Abbildung 5 ist das auf Ebene von Geschäftsmodellklassen gebildete Wertschöpfungsnetzwerk dargestellt. Diejenigen Geschäftsmodellklassen, für die sich in der Untersuchungsregion keine Geschäftsmodellprototypen identifizieren lassen, sind ausgegraut und treten somit außerhalb der Untersuchungsregion auf. Für die vorhandenen Geschäftsmodellklassen ist jeweils angegeben, wie viele dahinterstehende Prototypen insgesamt gemäß der Vollaufnahme von Giehl et al. (2020) existieren und wie viele Prototypen in der Untersuchungsregion identifiziert werden konnten. Für die Geschäftsmodellklasse Prosumer wird folgend davon ausgegangen, dass im untersuchten Raum mit hoher Wahrscheinlichkeit Verbraucher als Eigenstromproduzenten auftreten. Aufgrund des großen Erhebungsaufwandes werden die Häufigkeiten des Geschäftsmodells im Rahmen dieser Arbeit nicht quantifiziert. Das Geschäftsmodell des Prosumers wird nachfolgend als existierend angenommen. 

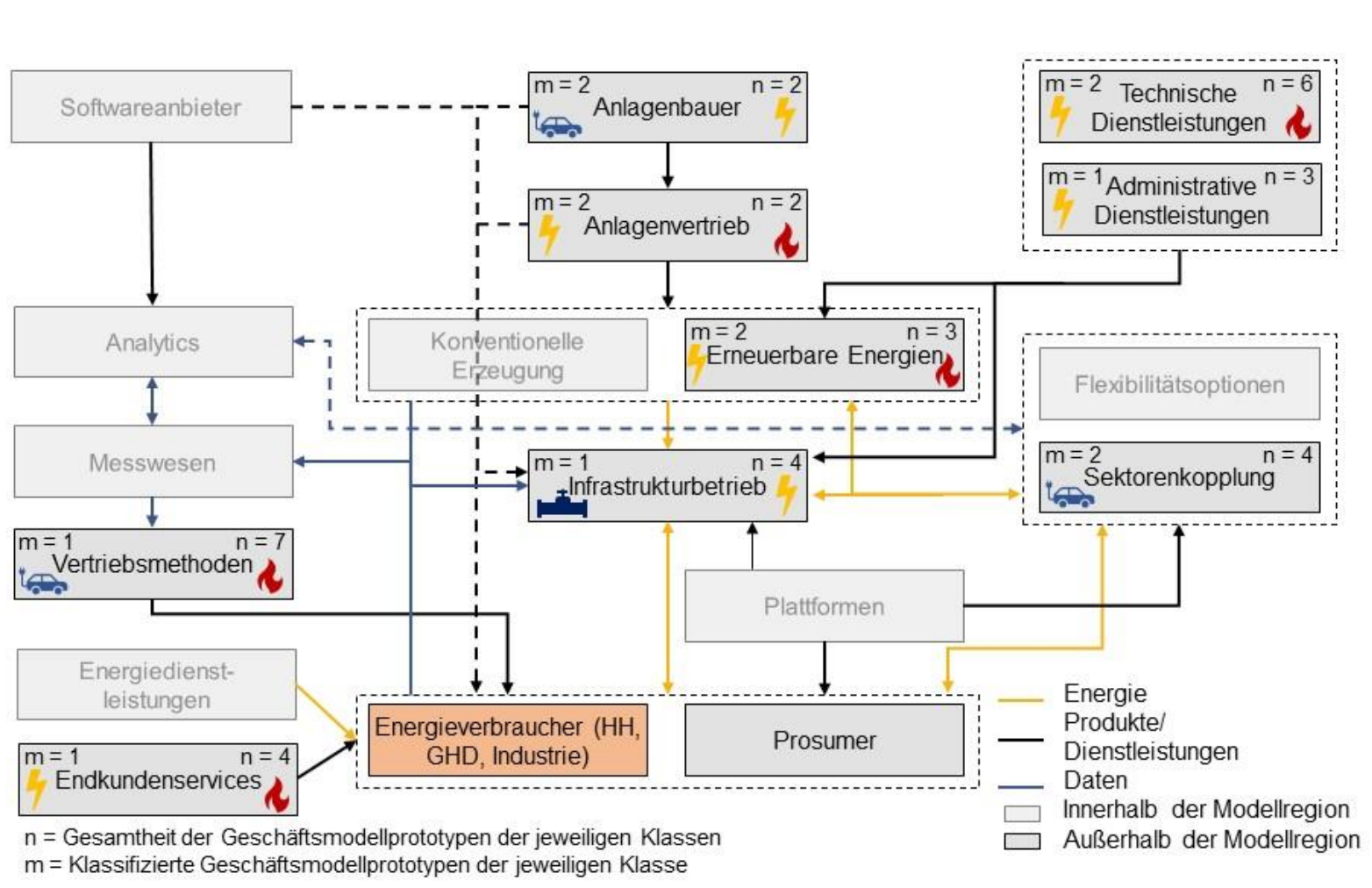

Abbildung 5: Identifizierte Geschäftsmodellklassen in der Modellregion Mecklenburg Quelle: Eigene Darstellung unter Verwendung von et al. (2020)

Deutlich wird im Netzwerk eine Dominanz von Asset-orientierten Leistungsinstanzen in der Modellregion. So sind beispielsweise die Klassen der Anlagenbauer, Anlagenvertriebe, Erneuerbaren Erzeugung, Infrastrukturbetrieb, Sektorenkopplung und Technischen Dienstleistungen alle an der materiellen Wertschöpfung vor Ort beteiligt, wobei es an immaterieller Wertschöpfung über Plattformen und Steuereinheiten eher mangelt. Durch das Fehlen der digitalen Klassen Analytics, Softwareanbieter und Plattformen ist festzustellen, dass fast der komplette Datenfluss im obigen Netzwerk zu regionenexternen Akteuren verläuft. Die Geschäftsmodelle der betroffenen Klassen sind somit in der Modellregion nicht anzutreffen. Ebenso sind Klassen, die ihr Geschäftsmodell auf das Endkundensegment ausrichten, wie das Messwesen oder Flexibilitätsoptionen, mit Ausnahme von Endkundenservices nicht repräsentiert.

\subsection{Entwicklung von regionalen prototypenbasierten und unternehmensspezifischen Wertschöpfungsnetzwerken}

Auf Grundlage der Zuordnung von Unternehmenstätigkeiten zu prototypischen Geschäftsmodellen wird ein Wertschöpfungsnetzwerk um einen repräsentativen Prototyp der Modellregion aufgebaut. Die Untersuchung ergab, wie in Kapitel 4.2 dargelegt, ein vermehrtes Auftreten der Prototypen Systemvertrieb, Endkundenvertrieb Energie und Erneuerbare Erzeugung. Da bei den Systemvertrieben das Leistungsspektrum jedoch häufig neben erneuerbaren Lösungen auch konventionelle Systeme wie Gas- und Ölheiztechnik beinhaltet, die nicht im Einklang mit der energiewendeförderlichen Ausrichtung des BMFE stehen, und der Endkundenvertrieb Energie ausschließlich aus Tankstellen besteht, wird das Wertschöpfungsnetzwerk auf Ebene von Geschäftsmodellprototypen für den Prototyp Erneuerbare Erzeugung aufgebaut. Erneut sind die nicht in der Untersuchungsregion vorhandenen Geschäftsmodellprototypen ausgegraut. Ergänzend ist für die regional vorhandenen Geschäftsmodellprototypen angegeben, wie häufig diese aufgefunden worden sind. 


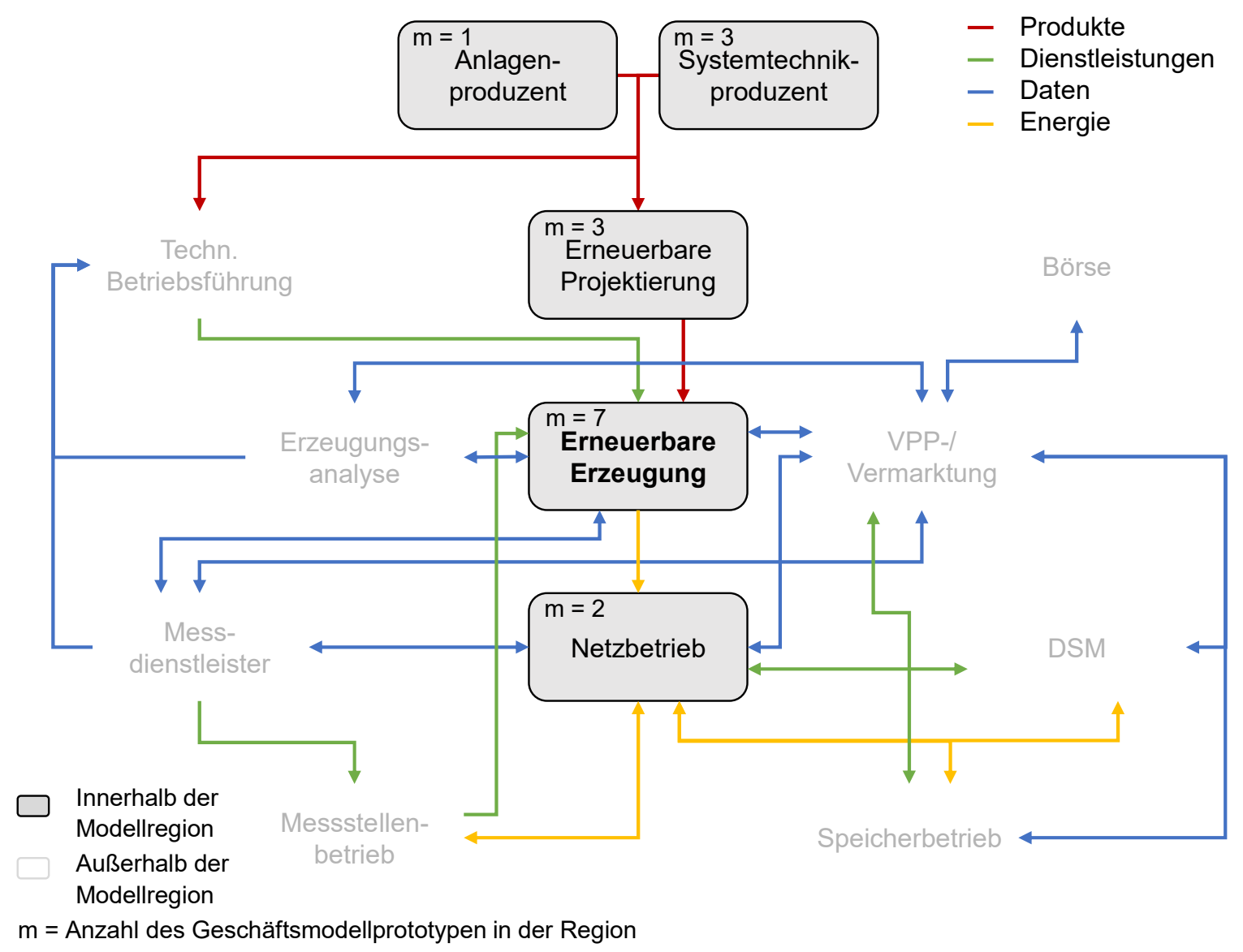

Abbildung 6. Das regionale Wertschöpfungsnetzwerk der Erneuerbaren Erzeugung

Quelle: Eigene Darstellung unter Verwendung von Giehl et al. 2020

Von dem abgebildeten prototypbasierten Netzwerk um den Prototyp Erneuerbare Erzeugung werden in der Untersuchungsregion nur wenige über das Wertschöpfungsnetzwerk verbundene Geschäftsmodellprototypen bedient. Es ist deutlich sichtbar, dass wertschöpfende Aktivitäten vorwiegend extern geschehen und damit nicht im regionalen Raum verbleiben. Die energiewirtschaftliche Wertschöpfung, die durch Erneuerbare Erzeugung erbracht wird, beschränkt sich dadurch räumlich nur auf einen sehr kleinen Anteil der Gesamtwertschöpfung des dargestellten Netzwerks. Lediglich der Produktfluss zur Bereitstellung der Anlagentechnologie wird von regionalen Unternehmen angeboten, wodurch die Konzentration Asset-orientierter Wertschöpfung auch hier deutlich wird. Dienstleistungen werden im oberen Netzwerk nicht von regionalen Unternehmen angeboten, was deutliche Wertschöpfungsdefizite der Region aufzeigt. Die nicht repräsentierten Geschäftsmodellprototypen Speicherbetrieb, Messstellenbetrieb sowie Technische Betriebsführung weisen regional durch lokale Standortvorteile große Potentiale auf und können vor allem Unterstützungsfunktionen für die vorhandenen Instanzen, wie dem Erzeuger oder dem Netzbetreiber, einnehmen. Sichtbar wird im prototypbasierten Netzwerk ebenfalls, dass der Datenaustausch des betrachteten Erzeugers nur extern stattfindet und weiterverarbeitet wird. 


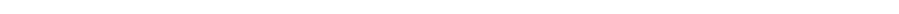

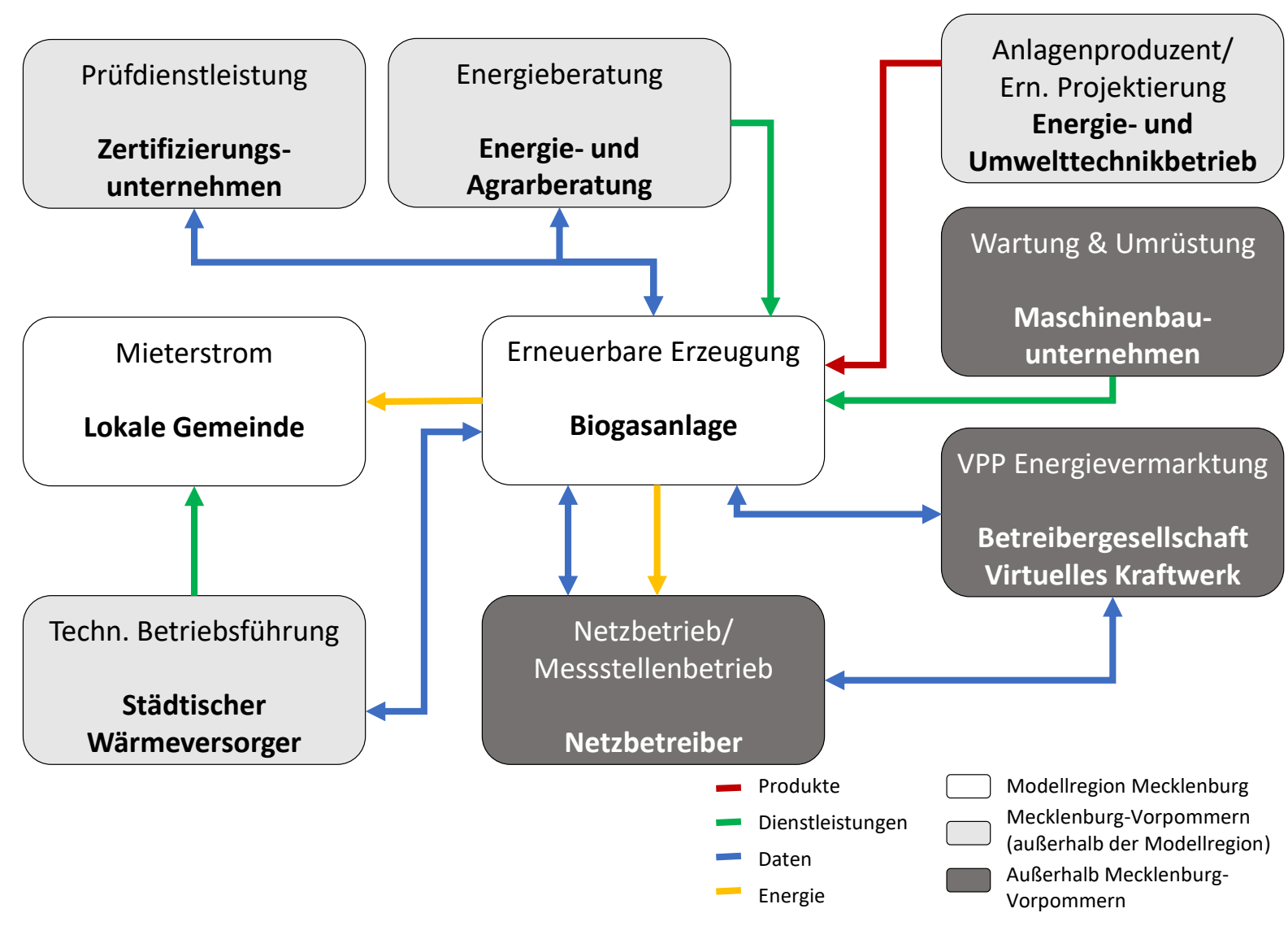

Abbildung 7: Wertschöpfungsnetzwerk für das betrachtete Beispielunternehmen

Quelle: Eigene Darstellung auf Basis eines Interviews mit dem analysierten Beispielunternehmen 2019

Im Rahmen der Fallstudie wird eine exemplarische Betreibergesellschaft einer Biogasanlage aus der Untersuchungsregion untersucht. Die Auswahl erfolgte zufällig für den repräsentativen Geschäftsmodellprototypen Erneuerbare Erzeugung mit dem Ziel, den aus der Theorie entwickelten Ansatz zur Bildung von Wertschöpfungsnetzwerken hinsichtlich realitätsnaher Anwendbarkeit zu überprüfen und somit zu validieren. Das Unternehmen speist Strom ins öffentliche Netz ein und vertreibt Wärme an die Standortgemeinde, welche als Wärmenetzbetreiber die Wärme durch ein autarkes System an die örtliche Bevölkerung vermarktet. Hierbei übernimmt ein weiteres Unternehmen im Auftrag der Gemeinde sowohl die technische als auch kaufmännische Betriebsführung des Wärmenetzes. Für den Haupttätigkeitsbereich des analysierten Unternehmens (Biogasanlagenbetreiber; Geschäftsmodellprototyp: Erneuerbare Erzeugung) wird ein Geschäftsmodellnetzwerk auf Ebene von Geschäftsmodellprototypen entwickelt, welches die zentralen Partner bzw. unmittelbar verbundenen Geschäftsmodellprototypen umfasst. Ferner ist durch farbliche Unterscheidung in Abbildung 7 kenntlich gemacht, welche Geschäftsmodellprototypen bzw. dahinterstehende Unternehmen innerhalb der Modellregion, innerhalb des Bundeslands MV bzw. außerhalb des Bundeslandes ansässig sind.

Im Vergleich zum aus der Theorie entwickelten Netzwerk in Abbildung 6 sind die direkten Partner für den Geschäftsmodellprototypen im realitätsnahen Beispiel in Abbildung 7 grundlegend dieselben. Zwar gibt es geringfügige Abweichungen, wie bspw. die beiden identifizierten Unternehmen für Prüfdienstleistungen bzw. für Energieberatung ${ }^{2}$ zeigen, allerdings können solche spezifischen Geschäftsmodellpartnerschaften je nach Technologie und spezifischer Geschäftsmodellausgestaltung variieren. Die realitätsnahe Überprüfung des aus der Theorie entwickelten Ansatzes zeigt erneut, dass nur ein

\footnotetext{
${ }^{2}$ Die eigentlich nur für den klassischen Endkundenservice ausgerichteten Geschäftsmodelle Prüfdienstleistungen und Energieberatung werden hier vom Erzeuger betriebsunterstützend in Anspruch genommen. Es ist eine Aufnahme des Erzeugers als Kunde für die allgemeinen Geschäftsmodellprototypen anzustreben.
} 
geringer Teil der wertschöpfenden Tätigkeiten in der Untersuchungsregion selbst auftritt und sich die kooperativen Unternehmensverflechtungen in weiten Teilen über die Region hinaus erstrecken.

\subsection{Ableitung von Handlungsoptionen für das Fallbeispiel}

Durch den regionalen Fokus auf erneuerbarer Energieerzeugung und die sich dahinter verbergenden Wertschöpfungspotenziale sind die Grundfaktoren zur Bildung von Wertschöpfungsclustern in der Modellregion Mecklenburg vorhanden. Zwei der wesentlichen vier Clusterbedingungen nach Porter, aufgeführt in Kapitel 2.2, sind in der Modellregion Mecklenburg bereits vorhanden: die Faktorbedingung in Form der spezifischen Standortvorteile erneuerbarer Energien und die Nachfragebedingung, welche durch die zunehmende Nachfrage nach erneuerbarem Strom im Zuge der Defossilisierung erfüllt wird. Die betrachteten Wertschöpfungsnetzwerke zeigen zwei Aspekte auf.

Zum einen ist die regionale Wirtschaft realgüterbasiert. Zum anderen ist die Energiewirtschaft auf das vorhandene Potenzial erneuerbarer Energien ausgerichtet. Es stellt sich somit die Frage, inwiefern die Standortvorteile der Energieerzeugung ausgeschöpft werden können, um kollaborative Wettbewerbsvorteile für die Region zu schaffen.

Die energiewirtschaftlichen Potenziale im Bereich der erneuerbaren Energien werden gut durch die in Kapitel 4.4 analysierten Wertschöpfungsnetzwerke deutlich. Sowohl auf Ebene der Geschäftsmodellprototypen als auch im Fall des betrachteten Beispielunternehmens eines Biogasanlagenbetreibers wird deutlich, dass die beteiligten Unternehmen nicht alle in der Untersuchungsregion anzutreffen sind. Vor diesem Hintergrund zeigt sich, dass mit einer Ansiedlung der entsprechenden Unternehmen Potenzial für die weitere wirtschaftliche Entwicklung gegeben sein sollte. Aufgrund der gegebener Größenvorteile einzelner Geschäftsmodellprototypen gilt dies nicht nur für die Untersuchungsregion. Ein Beitrag zur wirtschaftlichen Entwicklung kann auch schon damit erfüllt sein, dass sich entsprechende Unternehmen im Bundesland ansiedeln.

Basierend auf den vorhandenen und fehlenden Geschäftsmodell(prototyp)en, die die Wertschöpfungsnetzwerke aufzeigen, sind Defizite im Bereich datengetriebener als auch dienstleistungsgenerierender Geschäftsmodelle erkennbar. Geschäftsmodelle aus den Bereichen Energiedienstleistungen und Technische Dienstleistungen bieten regionalen Unternehmen die Möglichkeit, an dem vorhandenen Potenzial der erneuerbaren Energien zu partizipieren. Konkret die Technische Betriebsführung weist große Potentiale zum Nutzbarmachen der örtlichen Vernetzungsstrukturen auf. Indem sie Dritte aus der Region als Betriebsführer direkt in das örtliche Cluster einbindet, ist es möglich einen Beitrag regionale Wertschöpfung zu induzieren. Gleichermaßen sind Potenziale im Speicherbetrieb und in der Sektorenkopplung zu sehen, da Entwicklungsprojektionen für diese Bereiche insbesondere für sehr hohe Anteile erneuerbarer Energien eine zunehmende Relevanz voraussagen (vgl. Quaschning, 2016; FENES et al., 2014).

Den datengetriebenen Geschäftsmodellklassen wird dahingehend nur geringes Potential in der Modellregion zugesprochen. Unternehmen dieser Geschäftsmodelle operieren aus vernetzten Agglomerationen, die es ermöglichen, die Vorteile smarter innovativer Technik voll auszuschöpfen. Sie profitieren daher von komplett gegenteiligen Raumstrukturen. Für das Wertschöpfungscluster der Modellregion Mecklenburg bietet es sich daher an, die datengetriebenen Wertschöpfungsflüsse weiterhin extern zu beziehen und zu generieren. Um die datengetriebenen Geschäftsmodelle jedoch bestmöglich in das regionale Cluster einzubinden und Daten- sowie Informationsflüsse zu optimieren sind hier vor allem leistungsstarke Vernetzungsstrukturen notwendig.

Als unterstützende Branche wird deshalb vor allem der luK-Sektor gesehen. Der ländliche Raum weist häufig noch große Defizite bezüglich einer leistungsfähigen Breitbandversorgung auf, welche im Zuge der Digitalisierung als maßgebend für die Attraktivität von Lebens- und Wirtschafträumen zu sehen ist 
(BLE, 2014). Hier ist eine Verbesserung der Vernetzung für die Energieerzeugung und angrenzende Tätigkeiten im ländlichen Raum anzustreben.

\subsection{Limitationen der Analyse}

Mit Blick auf die Bestandsaufnahme und die Anwendung und Modifikation der Systematisierungen aus Giehl et al. (2020) ergeben sich Limitationen der Analyse. Dies bezieht sich in erster Linie auf die Menge der erfassten Unternehmen und Informationen. Ausgehend von der traditionellen Wertschöpfungskette und dem Fokus auf erneuerbare Energien ist es möglich, dass nicht alle energiewirtschaftlichen Unternehmen in der Region erfasst wurden. Anzumerken ist auch, dass die Größe der Untersuchungsregion Einfluss auf die Anzahl der gefundenen Geschäftsmodelle hat. Zudem ergibt sich aus der Nutzung von frei zugänglichen Daten, dass nicht zwangsläufig alle Informationen erfasst wurden. Der hohe Informationsbedarf zeigt sich beispielsweise beim Aufstellen von detaillierten Wertschöpfungsnetzwerken. Für ein konkretes Geschäftsmodell ist dies nur in Form einer detaillierten Fallstudie mit Zugang zu internen Unternehmensinformationen möglich.

Bezüglich der Zuordnung der betrachteten Unternehmen zu genau einem Geschäftsmodellprototypen ist zu beachten, dass dies aufgrund der gegebenen Größe der Unternehmen in der Region gut möglich war. Bei einer breit angelegten Geschäftstätigkeit ist es jedoch auch möglich, dass ein Unternehmen mehrere Geschäftsmodellprototypen realisiert. Mit Bezug auf die diskutierten Handlungsoptionen wäre eine Gegenüberstellung mit einer Vergleichsregion notwendig. Einerseits ließen sich weitergehende Feststellungen in Bezug auf regionale Wertschöpfungspotenziale ableiten. Andererseits wären weitergehende Aussagen in Bezug auf die Verallgemeinerbarkeit der Analysemethoden aus Giehl et al. (2020) möglich.

\section{Fazit und Ausblick}

Ziel der Analysen in diesem Papier war es, einerseits eine Bestandsaufnahme der regionalen energiewirtschaftlichen Wertschöpfung in der Untersuchungsregion Modellregion Mecklenburg durchzuführen. Andererseits sollten die grundlegenden methodischen Ansätze aus Giehl et al. (2020) und Giehl et al. (2019) auf die kleinräumige Untersuchungsregion übertragen sowie anhand eines praktischen Fallbeispiels validiert werden. Ferner sollen hieraus Handlungsempfehlungen für energiewirtschaftliche Wertschöpfungspotenziale abgeleitet werden. Nachfolgend sollen zunächst die zentralen Erkenntnisse der Analyse auch vor dem Hintergrund der im vorigen Kapitel beschriebenen Limitationen zusammengefasst und abschließend ein Forschungsausblick gegeben werden.

\subsection{Zusammenfassung}

Die Bestandsaufnahme der energiewirtschaftlichen Wertschöpfungskette für die Modellregion Mecklenburg liefert im Ergebnis 33 relevante Tätigkeiten für den Landkreis NWM und 29 für die MSE. Schwerpunkte zeigen sich in den Wertschöpfungsbereichen Herstellung und Errichtung von Anlagen, technische Betriebsführung sowie Vertrieb. Windenergie- und Biomasseanlagen sowie entsprechende Wertschöpfungsbeiträge sind - wenngleich in ausbaufähigem Umfang - in der Untersuchungsregion vorhanden.

Basierend auf der Erfassung relevanter Unternehmen wird eine Zuordnung zu Geschäftsmodellprototypen durch Erfassung der der Ausprägungen der Komponenten des BMFE vorgenommen. Im Ergebnis können alle regionalen Unternehmen genau einem vorhandenen Geschäftsmodellprototypen aus der Klassifizierung nach Giehl et al. (2019 und 2020) zugeordnet werden. Somit wird die Vollständigkeit der umfassenden Momentaufnahme der energiewirtschaftlichen Geschäftsmodelle - zumindest für die betrachtete Untersuchungsregion - bestätigt. Es werden lediglich geringfügige Anpassungen und Ergänzungen bei einzelnen Komponentenausprägungen für einzelne Geschäftsmodellprototypen des BMFE, insbesondere bei der Komponente Technologie, empfohlen. 
Das Analyseinstrument des Wertschöpfungsnetzwerks wird erfolgreich auf regionaler Ebene angewendet. Hierbei werden kleinere methodische Erweiterungen in Bezug auf die geografische Zuordnung, die Information der abgedeckten Sektoren (Strom, Wärme, Gas, Verkehr) sowie den Stichprobenumfang eingeführt. Das regionale Wertschöpfungsnetzwerk wird zunächst auf Ebene von Geschäftsmodellklassen für die Untersuchungsregion entwickelt, wobei sich Abdeckungslücken in Bezug auf einige Geschäftsmodellklassen bzw. -prototypen, insbesondere solche, die mit digitalen Technologien oder Dienstleistungen einhergehen, feststellen lassen. Ferner wird ein Geschäftsmodellnetzwerk auf Ebene von Geschäftsmodellprototypen zunächst aus der Theorie für die Erneuerbare Erzeugung als repräsentativer Geschäftsmodellprototyp für die Untersuchungsregion entwickelt und nachfolgend seine Übertragbarkeit auf ein reales Beispielunternehmen demonstriert. Hierbei sind erneut für die Region Abdeckungslücken in Bezug auf angrenzende Services festzustellen.

Zuletzt werden Wertschöpfungspotentiale eines regionalen Wertschöpfungsclusters rund um den Geschäftsmodellprototyp Erneuerbare Erzeugung der Modellregion Mecklenburg in der Nutzbarmachung der ländlichen Gegebenheiten identifiziert. Als Faktorbedingungen werden dabei Standortvorteile der erneuerbaren Energieerzeugung in Kombination mit einer zunehmenden Nachfrage nach erneuerbarem Strom im Zuge der Energiewende identifiziert, sodass zwei der vier Bestimmungsfaktoren nach Porter (1991) bereits gegeben sind. Die Chancen und Herausforderungen des ländlichen Raums vor allem in der Verbesserung des örtlichen Wettbewerbs und einer differenzierteren strategischer Ausrichtung der Unternehmen im Rahmen der Wertschöpfungsnetzwerke zur Erfüllung der beiden verbleibenden Bestimmungsfaktoren für Wertschöpfungscluster nach Porter (1991). Vor allem Geschäftsmodelle mit realgüterwirtschaftlichem und dienstleistungsfokussiertem Hintergrund versprechen die größten Möglichkeiten an der erneuerbaren Erzeugung zu partizipieren. In der Modellregion bestehen hinsichtlich der Akteursvielfalt und -diversität dieser Geschäftsmodellklassen noch große Ausbaumöglichkeiten, wie die geringen Stichprobenumfänge der vertretenden Prototypen zeigen. Für eine genauere Untersuchung von Wertschöpfungsstrukturen und -potenzialen empfiehlt es sich daher, prototypbasierte Netzwerke in die Betrachtung aufzunehmen. Das gegenüber dem klassenbasierten Ansatz detaillierte Verfahren bietet die Möglichkeit, komplexere und spezifischere Verflechtungen auf den Teilbereichen des Energiesektors aufzustellen. Die Fallstudie zeigt ferner, dass durch die vordefinierten BMFE-Komponenten- und Prototypenausprägungen reale Unternehmenswertschöpfungsnetzwerke abgebildet werden, die inner- und zwischenbetriebliche Verflechtungen aufzeigen können und als Analyseinstrument für (betriebsinterne) einzelökonomische Untersuchungen herangezogen werden können. Den in den Wertschöpfungsnetzwerken fehlenden datengetriebenen Geschäftsmodellen wird in der Modellregion nur geringfügiges Potential aufgrund der gegebenen Raumstrukturen zugesprochen. Hier sollte der Fokus auf dem Ausbau der Infrastruktur für unterstützende luK-Branche liegen, um die Daten- und Informationsflüsse sowohl zu den externen datengetriebenen Wertschöpfungsinstanzen als auch innerhalb des Clusters zu verbessern.

\subsection{Ausblick}

Anknüpfender Forschungsbedarf besteht hinsichtlich einer Modifikation der Erhebungsmethodik zur Identifikation energiewirtschaftlicher Wertschöpfung. Hierbei könnten Wertschöpfungsnetzwerke anstelle von Wertschöpfungsketten als Ausgangspunkt der Analysen gewählt werden. Ferner könnten auch nicht öffentlich verfügbare Quellen zum Schließen von Datenlücken herangezogen werden.

Die Analysen zeigen, dass sich das BMFE zwar für die Untersuchungsregion als robustes und weitgehend vollständiges Analyseelement erwiesen hat. Dennoch wird der Charakter eines dynamisch erweiterbaren Analyseinstruments durch die wenigen empfohlenen Modifikationen und Erweiterung deutlich. Die Vermutung liegt nahe, dass bei einer Anwendung in weiteren Untersuchungsregionen weitere Anpassungs- und Ergänzungsbedarfe zu Tage treten könnten. 
Auch zur Absicherung der Robustheit besteht weitergehender Forschungsbedarf in der Übertragung der hiesigen Analysesystematik auf weitere Betrachtungs- und Vergleichsregionen, nicht zuletzt, um die Belastbarkeit von Handlungsempfehlungen in Bezug auf regionale energiewirtschaftliche Wertschöpfungspotenziale zu steigern.

Ferner besteht Forschungsbedarf in einer Ergänzung der überwiegend qualitativen Analyseinstrumente um eine quantitative (monetäre) Bewertung von Zahlungsströmen (im Wesentlichen Erlös- und Kostenpositionen) für Geschäftsmodelle bzw. Geschäftsmodellprototypen. Analysen zur Entwicklung von qualitativen und quantitativen Bewertungsansätzen zur Bewertung des Erfolgspotenzials von Geschäftsmodellprototypen werden derzeit am Fachgebiet für Energie- und Ressourcenmanagement der Technischen Universität Berlin erarbeitet.

\section{Danksagung und Förderhinweis}

Der vorliegende Artikel ist im Rahmen des durch das Bundesministerium für Bildung und Forschung (BMBF) geförderten Kopernikus-Projekts "Systemintegration": Energiewende-Navigationssystem (ENavi) (Förderkennzeichen 03SFK4NO) entstanden. Die Autorinnen und Autoren möchten sich für die Finanzierung der Arbeiten bedanken.

\section{Literatur}

Bach, N. \& Krüger, W. (2003). Geschäftsmodelle für Wertschöpfungsnetzwerke: Wilfried Krüger zum 60. Geburtstag., 1. Aufl Wiesbaden 2003.

Baur, C. \& Kluge, J. (2000). Die Wertkette als Instrument der strategischen Analyse. in: Praxis des Strategischen Managements, hrsgg. v. M. K. Welge, A. Al-Laham \& P. Kajüter, Wiesbaden 2000, S. 135-146, URL: http://www.springerlink.com/index/10.1007/978-3-663-05965-3_7, 08.06.2017.

BBSR (2012). Raumordnungsbericht 2011. 2012, URL: http://www.bbsr.bund.de/BBSR/DE/Veroeffentlichungen/Sonderveroeffentlichungen/2012/DL_ROB2011.pdf;jsessio-

nid=437E52F5CA530A2C50A7EF7739AB0F70.live11291?_blob=publicationFile\&v=2, 20.12.2019.

Bieger, T. \& Reinhold, S. (2011). Das wertbasierte Geschäftsmodell - Ein aktualisierter Strukturierungsansatz. in: Innovative Geschäftsmodelle, hrsgg. v. T. Bieger, D. zu Knyphausen-Aufseß \& C. Krys, Berlin, Heidelberg 2011, S. 13-70, URL: http://link.springer.com/10.1007/978-3-642-180682_2, 19.08.2019.

Biem, A. \& Caswell, N. (2008). A Value Network Model for Strategic Analysis. 2008, S. 361-361, URL: https://ieeexplore.ieee.org/document/4439066/, 19.08.2019.

BLE, B. für L. und E. (2014). Nutzungschancen des Breitbandinternets für ländliche Räume: Innovative Anwendungen, neue Ideen, gute Beispiele. 2014, URL: http://link.springer.com/10.1007/BF03161389, 09.07.2019.

BMVBS, B. für V., Bau und Stadtentwicklung (2011). Erneuerbare Energien: Zukunftsaufgabe der Regionalplanung. Berlin 2011, URL: http://beschaffung.fnr.de/fileadmin/nawaro-kommunal/dateien/DL_ErneuerbareEnergien.pdf, 15.12.2018.

Demil, B. \& Lecocq, X. (2010). Business Model Evolution: In Search of Dynamic Consistency. Long Range Planning, 2-3 (43), S. 227-246. 
Destatis (2019). Bruttowertschöpfung. 2019, URL: https://www.destatis.de/DE/Themen/Wirtschaft/Volkswirtschaftliche-Gesamtrechnungen-Inlandsprodukt/Glossar/bruttowertschoepfung.html, 14.08.2019.

Dyckhoff, H. (2003). Grundzüge der Produktionswirtschaft: Einführung in die Theorie betrieblicher Wertschöpfung., 4., verb. Aufl. Berlin 2003.

FENES, IAEW, ef.Ruhr \& ISEA (2014). Strompreicher in der Energiewende. Untersuchung zum Bedarf an neuen Stromspeichern in Deutschland für den Erzeugungsausgleich, Systemdienstleistungen und im Verteilnetz., URL: https://speicherinitiative.at/assets/Uploads/19-AgoraEnergiewende-Speicherstudie-Langfassung.pdf, 14.06.2019.

Gabler Wirtschaftslexikon (2019). Definition: Wertschöpfung., URL: https://wirtschaftslexikon.gabler.de/definition/wertschoepfung-50306, 03.02.2019.

GWS, Gesellschaft für Wirtschaftliche Strukturforschung mbH (2016). Gesamtwirtschaftliche Wirkungen der Exporttätigkeit der deutschen PV- und Windindustrie. Der Aufbau internationaler Wertschöpfungsketten und Deutschlands Rolle auf dem Weltmarkt für Technologien zur Nutzung erneuerbarer Energien. GWS Research Report, URL: http://www.gws-os.com/discussionpapers/gws-researchreport16-2.pdf, 10.05.2018.

Giehl, J. F., Göcke, H., Grosse, B., Kochems, J., v. Mikulicz-Radecki, F. \& Müller-Kirchenbauer, J. (2019). Data Documentation Energie und Ressourcen: Vollaufnahme und Klassifikation von Geschäftsmodellen der Energiewende. Zenodo, URL: https://zenodo.org/record/3518997, 19.11.2019.

Giehl, J. F., Göcke, H., Grosse, B., Kochems, J. \& Müller-Kirchenbauer, J. (2020). V Survey and Classification of Business Models for the Energy Transformation. Energies, 13(11). 2981. https://doi.org/10.3390/en13112981

Haller, A. (1997). Wertschöpfungsrechnung : Ein Instrument zur Steigerung der Aussagefähigkeit von Unternehmensabschlüssen im internationalen Kontext. Stuttgart 1997.

Handelsregister (2019). Gemeinsames Registerportal der Länder. 2019, URL: https://www.handelsregister.de/rp_web/welcome.do.

Landkreis Mecklenburgische Seenplatte (2019). Geoportal Landkreis Mecklenburgische Seenplatte. 2019, URL: https://geoport-lk-mse.de/geoportal/index.php.

Landkreis Nordwestmecklenburg (2019). Geoportal Nordwestmecklenburg. 2019, URL: https://www.geoport-nwm.de/de/energie.html.

Landtag Mecklenburg-Vorpommern (2018). KLEINE ANFRAGE der Abgeordneten Dr. Mignon Schwenke,Fraktion DIE LINKE, Windkraftanlagen in Mecklenburg-Vorpommern, Drucksache7/1653. 2018, URL: https://s3.kleine-anfragen.de/ka-prod/mv/7/1653.pdf.

Mayring, P. (2016). Einführung in die qualitative Sozialforschung: eine Anleitung zu qualitativem Denken., 6. Auflage Weinheim 2016, URL: http://bvbr.bib-bvb.de:8991/F?func=service\&doc_library=BVB01\&local_base $=$ BVB01\&doc_number $=028771494 \&$ sequence $=000002 \&$ line_number $=0001 \&$ func_code=DB_RECORDS\&service_type=MEDIA, 24.01.2019. 
Meffert, H. (1989). Die Wertkette als Instrument einer integrierten Unternehmensplanung. in: Der Integrationsgedanke in der Betriebswirtschaftslehre, hrsg. v. W. Delfmann, Wiesbaden 1989, S. 255-278, URL: http://link.springer.com/10.1007/978-3-322-83652-6_13, 08.06.2017.

Ministerium für Landwirtschaft und Umwelt (2019). Biomasseanlagen in Mecklenburg-Vorpommern. 2019, URL: https://www.regierung-mv.de/Landesregierung/Im/Umwelt/Immissionsschutz/Biomasseanlagen-in-MV/.

Miroschedji, S. A. de (2002). Globale Unternehmens- und Wertschöpfungsnetzwerke: Grundlagen, Organisation, Gestaltung., 1. Aufl. Wiesbaden 2002, URL: http://bvbr.bibbvb.de:8991/F?func=service\&doc_library=BVB01\&local_base=BVB01\&doc_number $=010022299 \&$ sequence $=000002 \&$ line_number $=0001 \&$ func_code $=D B \_R E C O R D S \&$ service_type=MEDIA, 07.02.2019.

Netztransparenz.de (2018). EEG-Anlagenstammdaten 50Hertz Transmission GmbH zur Jahresabrechnung 2018. 2018.

Osterwalder, A. \& Pigneur, Y. (2010). Business Model Generation: A Handbook for Visionaries, Game Changers, and Challengers. Hoboken, New Jersey 2010, URL: http://ebookcentral.proquest.com/lib/tuberlin/detail.action?docID=581476, 02.01.2019.

Peppard, J. \& Rylander, A. (2006). From Value Chain to Value Network: European Management Journal, 2-3 (24), S. 128-141.

Porter, M. E. (1985). TECHNOLOGY AND COMPETITIVE ADVANTAGE. Journal of Business Strategy, 3 (5), S. $60-78$.

Porter, M. E. (1991). Nationale Wettbewerbsvorteile: erfolgreich konkurrieren auf dem Weltmarkt. München 1991.

Quaschning, V. (2016). Sektorkopplung durch die Energiewende. , S. 38.

Statistisches Bundesamt (2018). Zahlen und Fakten - Länder \& Regionen - Alle politisch selbstständigen Gemeinden mit ausgewählten Merkmalen am 31.03.2018 (1. Quartal). 2018, URL: https://www.destatis.de/DE/ZahlenFakten/LaenderRegionen/Regionales/Gemeindeverzeichnis/Administrativ/Archiv/GVAuszugQ/AuszugGV1QAktuell.xlsx;jsessionid=36424EDE9D3D0494AF585297473EFA54. InternetLive2?_blob=publicationFile., 10.05.2018.

Steinle, C. \& Schiele, H. (2010). Die räumliche Dimension im Strategischen Management von Wertschöpfungssystemen: Operationalisierung des Cluster - Ansatzes für die strategische Analyse. in: Geschäftsmodelle für Wertschöpfungsnetzwerke - Begriffliche und konzeptionelle Grundlagen, hrsgg. v. N. Bach, W. Buchholz \& B. Eichler, Wiesbaden, 2. Auflage 2010.

Teece, D. J. (2010). Business Models, Business Strategy and Innovation. Long Range Planning, 2-3 (43), S. 172-194.

Weber, W., Kabst, R. \& Baum, M. (2018). Einführung in die Betriebswirtschaftslehre., 10. Aufl. Wiesbaden 2018, URL: https://doi.org/10.1007/978-3-658-18252-6, 14.03.2019.

(2011). Neue Energie im Osten - Gestaltung des Umbruchs: Perspektiven für eine zukunftsfähige sozial-ökologische Energiewende. Frankfurt am Main [u.a.] 2011. 


\section{Anhang}

8.1 Anhang 1: Geschäftsmodellklassifizierung der Modellregion Mecklenburg

Tabelle 1: Geschäftsmodellklassifizierung regionaler Unternehmen Quelle: Eigene Darstellung unter Verwendung von Giehl et al. 2019

\begin{tabular}{|c|c|c|c|c|c|c|c|}
\hline Unternehmen & Nutzenversprechen & Kundensegment & $\begin{array}{l}\text { Ertrags- } \\
\text { modell }\end{array}$ & $\begin{array}{l}\text { Wertschöpf.- } \\
\text { stufe }\end{array}$ & Partner & Technologie & $\begin{array}{l}\text { Geschäftsmodell- } \\
\text { prototyp }\end{array}$ \\
\hline $\begin{array}{l}\text { Unselbstst ndige } \\
\text { Tankstelle } \\
\text { (Total) }\end{array}$ & $\begin{array}{l}\text { Kraftstoff- } \\
\text { bereitstellung, } \\
\text { Mobilität }\end{array}$ & Autonutzer & Handelsgewinne & $\begin{array}{l}\text { Vertrieb, } \\
\text { Handel, } \\
\text { Mobilität }\end{array}$ & $\begin{array}{l}\text { Messdienstleister, Erzeuger, } \\
\text { Abrechnungsdienstleister, } \\
\text { Händler, Datenverarbeiter }\end{array}$ & $\begin{array}{l}\text { Mess- und } \\
\text { Steuerungstechnik }\end{array}$ & $\begin{array}{l}\text { Endkunden- } \\
\text { vertrieb Energie }\end{array}$ \\
\hline Stromtankstelle & $\begin{array}{l}\text { Strom, Mobilität, } \\
\text { Anlagenbetrieb, } \\
\text { Marktzugang, } \\
\text { Abrechnung }\end{array}$ & Autonutzer & $\begin{array}{l}\text { Nutzungs- } \\
\text { gebühren, } \\
\text { Servicegebühren, } \\
\text { Provision, } \\
\text { Asset-Verkauf }\end{array}$ & $\begin{array}{l}\text { Vertrieb, } \\
\text { Mobilität, } \\
\text { Service }\end{array}$ & $\begin{array}{l}\text { Technologieanbieter, } \\
\text { Messdienstleister, } \\
\text { Softwareanbieter, } \\
\text { Automobilhersteller, } \\
\text { Datenplattform }\end{array}$ & $\begin{array}{l}\text { Ladeinfrastruktur, } \\
\text { Software, Mess- } \\
\text { und Steuerungs- } \\
\text { technik }\end{array}$ & $\begin{array}{l}\text { Power-to- } \\
\text { Mobility-Lade- } \\
\text { infrastruktur }\end{array}$ \\
\hline PMC GmbH & $\begin{array}{l}\text { Anlagen für } \\
\text { Energietransport/ } \\
\text { Energievertrieb, } \\
\text { Energiespeicher }\end{array}$ & $\begin{array}{l}\text { Mobilitätsservice- } \\
\text { anbieter, Erzeuger, } \\
\text { Speicherbetreiber, } \\
\text { HH, GHD, EVU }\end{array}$ & Asset-Verkauf & $\begin{array}{l}\text { Technologie- } \\
\text { anbieter }\end{array}$ & $\begin{array}{l}\text { Technologieanbieter, } \\
\text { Mobilitätsserviceanbieter }\end{array}$ & $\begin{array}{l}\text { Mess- und } \\
\text { Steuerungstechnik }\end{array}$ & $\begin{array}{l}\text { Anlagen- } \\
\text { produzent }\end{array}$ \\
\hline $\begin{array}{l}\text { AWEK Protech } \\
\text { GmbH }\end{array}$ & $\begin{array}{l}\text { Anlagen- } \\
\text { komponenten }\end{array}$ & $\begin{array}{l}\text { Anlagenbauer, } \\
\text { Industrie, EVU, } \\
\text { Versorger, GHD }\end{array}$ & Asset-Verkauf & $\begin{array}{l}\text { Technologie- } \\
\text { anbieter }\end{array}$ & $\begin{array}{l}\text { Technologieanbieter, } \\
\text { Anlagenbetreiber, EVU, } \\
\text { Projektierer }\end{array}$ & $\begin{array}{l}\text { Mess- und } \\
\text { Steuerungstechnik }\end{array}$ & $\begin{array}{l}\text { Systemtechnik- } \\
\text { produzent }\end{array}$ \\
\hline $\begin{array}{l}\text { Johannovsky } \\
\text { Hydraulik } \\
\text { GmbH }\end{array}$ & $\begin{array}{l}\text { Anlagen- } \\
\text { komponenten }\end{array}$ & $\begin{array}{l}\text { Anlagenbauer, } \\
\text { Industrie, EVU, } \\
\text { Versorger, GHD }\end{array}$ & Asset-Verkauf & $\begin{array}{l}\text { Technologie- } \\
\text { anbieter }\end{array}$ & $\begin{array}{l}\text { Technologieanbieter, } \\
\text { Anlagenbetreiber, EVU, } \\
\text { Projektierer }\end{array}$ & $\begin{array}{l}\text { Mess- und } \\
\text { Steuerungstechnik, } \\
\text { Prüftechnik }\end{array}$ & $\begin{array}{l}\text { Systemtechnik- } \\
\text { produzent }\end{array}$ \\
\hline
\end{tabular}




\begin{tabular}{|c|c|c|c|c|c|c|c|}
\hline $\begin{array}{l}\text { Umweltscho- } \\
\text { nende Heiz- und } \\
\text { Energiesysteme } \\
\text { Ing. Reinhard } \\
\text { Schulz }\end{array}$ & $\begin{array}{l}\text { Klimaschutzbeitrag, } \\
\text { Erneuerbare Wärme } \\
\text { \& Strom, } \\
\text { Energieautarkie, } \\
\text { Komplettlösung - } \\
\text { Planung, Installation, } \\
\text { Wartung }\end{array}$ & $\begin{array}{l}\text { Haushalte, GHD, } \\
\text { Öffentl. Unternehmen }\end{array}$ & $\begin{array}{l}\text { Servicegebühren, } \\
\text { Asset-Verkauf }\end{array}$ & $\begin{array}{l}\text { Wohnen, } \\
\text { Service }\end{array}$ & $\begin{array}{l}\text { Technologieanbieter, } \\
\text { Anlagenbetreiber, } \\
\text { lokales Handwerk }\end{array}$ & $\begin{array}{l}\text { Solarthermie, } \\
\text { Photovoltaik-Klein, } \\
\text { BHKW, Biomasse- } \\
\text { anlagen, Energie- } \\
\text { speichertechnolo- } \\
\text { gien, Haustechnik, } \\
\text { Heiztechnik }\end{array}$ & Systemvertrieb \\
\hline $\begin{array}{l}\text { Heizung Sanitär } \\
\text { Nicky Rudolf }\end{array}$ & $\begin{array}{l}\text { Erneuerbare Wärme, } \\
\text { Wohnkomfort, Kom- } \\
\text { plettlösung - Planung, } \\
\text { Installation, Wartung }\end{array}$ & $\begin{array}{l}\text { Haushalte, GHD, } \\
\text { Öffentl. Unternehmen }\end{array}$ & $\begin{array}{l}\text { Servicegebühren, } \\
\text { Asset-Verkauf }\end{array}$ & $\begin{array}{l}\text { Wohnen, } \\
\text { Service }\end{array}$ & $\begin{array}{l}\text { Technologieanbieter, } \\
\text { Anlagenbetreiber, } \\
\text { lokales Handwerk }\end{array}$ & $\begin{array}{l}\text { Solarthermie, } \\
\text { Wärmepumpen, } \\
\text { Heiztechnik }\end{array}$ & Systemvertrieb \\
\hline $\begin{array}{l}\text { Geb ude- } \\
\text { reinigung } \\
\text { Burmeister \& } \\
\text { Spill GmbH }\end{array}$ & Anlagenwartung & Erzeuger & Servicegebühren & Service & $\begin{array}{l}\text { Technologieanbieter, } \\
\text { Anlagenbetreiber }\end{array}$ & keine & $\begin{array}{l}\text { Wartung und } \\
\text { Umrüstung }\end{array}$ \\
\hline $\begin{array}{l}\text { WindMW } \\
\text { GmbH }\end{array}$ & $\begin{array}{l}\text { Schlüsselfertige } \\
\text { erneuerbare Energie- } \\
\text { erzeugungsanlagen - } \\
\text { Projektplanung, } \\
\text {-umsetzung, } \\
\text {-finanzierung }\end{array}$ & EVU & $\begin{array}{l}\text { Servicegeb hren, } \\
\text { Asset-Verkauf }\end{array}$ & $\begin{array}{l}\text { Service, } \\
\text { Erzeugung }\end{array}$ & $\begin{array}{l}\text { Technologieanbieter, } \\
\text { Baugewerbe, Behörden, } \\
\text { Netzbetreiber, Anlagen- } \\
\text { betreiber, Finanzierer, } \\
\text { Projektpartner, Anlagen- } \\
\text { vertrieb, lokales Handwerk }\end{array}$ & $\begin{array}{l}\text { Große erneuerbare } \\
\text { Erzeuger }\end{array}$ & $\begin{array}{l}\text { Erneuerbare } \\
\text { Projektierung }\end{array}$ \\
\hline $\begin{array}{l}\text { integrity MS2 } \\
\text { GmbH }\end{array}$ & $\begin{array}{l}\text { Projektplanung, } \\
\text {-umsetzung, } \\
\text { Administration }\end{array}$ & $\begin{array}{l}\text { Erzeuger, Energie- } \\
\text { genossenschaften, } \\
\text { EVU }\end{array}$ & $\begin{array}{l}\text { Servicegeb hren, } \\
\text { Asset-Verkauf }\end{array}$ & Service & $\begin{array}{l}\text { Behörden, Baugewerbe, } \\
\text { Projektpartner, Netzbetreiber, } \\
\text { Technologieanbieter, } \\
\text { Anlagenbetreiber }\end{array}$ & $\begin{array}{l}\text { Große erneuerbare } \\
\text { Erzeuger }\end{array}$ & $\begin{array}{l}\text { Erneuerbare } \\
\text { Projektierung }\end{array}$ \\
\hline
\end{tabular}




\begin{tabular}{|c|c|c|c|c|c|c|c|}
\hline Kropf \& Bendlin & $\begin{array}{l}\text { Erneuerbare Wärme } \\
\& \text { Strom, } \\
\text { Komplettlösung - } \\
\text { Planung, Installation, } \\
\text { Wartung; } \\
\text { Wohnkomfort }\end{array}$ & $\begin{array}{l}\text { HH, GHD, Öffentl. } \\
\text { Unternehmen, } \\
\text { Wohnungsbau- } \\
\text { gesellschaften }\end{array}$ & $\begin{array}{l}\text { Servicegebühren, } \\
\text { Asset-Verkauf }\end{array}$ & $\begin{array}{l}\text { Wohnen, } \\
\text { Service }\end{array}$ & $\begin{array}{l}\text { Technologieanbieter, } \\
\text { Anlagenbetreiber, } \\
\text { lokales Handwerk }\end{array}$ & $\begin{array}{l}\text { Photovoltaik-Klein, } \\
\text { Solarthermie, } \\
\text { Biomasseanlagen, } \\
\text { BHKW, KWK, } \\
\text { Wärmepumpen, } \\
\text { Haustechnik }\end{array}$ & Systemvertrieb \\
\hline Sven G nther & $\begin{array}{l}\text { Erneuerbare Wärme, } \\
\text { Komplettlösung - } \\
\text { Beratung, Planung, } \\
\text { Installation, Wartung }\end{array}$ & $\begin{array}{l}\mathrm{HH}, \mathrm{GHD} \text {, } \\
\text { Öffentl. Unternehmen }\end{array}$ & $\begin{array}{l}\text { Servicegeb hren, } \\
\text { Asset-Verkauf }\end{array}$ & $\begin{array}{l}\text { Wohnen, } \\
\text { Service }\end{array}$ & $\begin{array}{l}\text { Technologieanbieter, } \\
\text { Anlagenbetreiber, } \\
\text { lokales Handwerk }\end{array}$ & $\begin{array}{l}\text { Solarthermie, } \\
\text { Wärmepumpen, } \\
\text { Heiztechnik }\end{array}$ & Systemvertrieb \\
\hline $\begin{array}{l}\text { Biogas } \\
\text { Wakenstädt } \\
\text { GbR }\end{array}$ & $\begin{array}{l}\text { Erneuerbarer Strom, } \\
\text { Klimaschutzbeitrag }\end{array}$ & $\begin{array}{l}\text { HH, GHD, Industrie, } \\
\text { Netzbetreiber, EVU, } \\
\text { H ndler, St dte und } \\
\text { Kommunen, ffentl. } \\
\text { Unternehmen }\end{array}$ & $\begin{array}{l}\text { Einspeise- } \\
\text { verg tung, } \\
\text { Grundgebühr, } \\
\text { Energiepreis }\end{array}$ & Erzeugung & $\begin{array}{l}\text { Netzbetreiber, Vermarkter, } \\
\text { Messstellenbetreiber, } \\
\text { Technologieanbieter, } \\
\text { EVU, Landwirtschaft }\end{array}$ & $\begin{array}{l}\text { Große erneuerbare } \\
\text { Erzeuger }\end{array}$ & $\begin{array}{l}\text { Erneuerbare } \\
\text { Erzeugung }\end{array}$ \\
\hline $\begin{array}{l}\text { HSE } \\
\text { Haustechnik }\end{array}$ & $\begin{array}{l}\text { Erneuerbare Wärme, } \\
\text { Wohnkomfort, } \\
\text { Komplettlösung - Be- } \\
\text { ratung, Planung, In- } \\
\text { stallation, Wartung }\end{array}$ & $\begin{array}{l}\mathrm{HH}, \mathrm{GHD}, \\
\text { ffentl. Unternehmen }\end{array}$ & $\begin{array}{l}\text { Servicegeb hren, } \\
\text { Asset-Verkauf }\end{array}$ & $\begin{array}{l}\text { Wohnen, } \\
\text { Service }\end{array}$ & $\begin{array}{l}\text { Technologieanbieter, } \\
\text { Anlagenbetreiber, } \\
\text { lokales Handwerk }\end{array}$ & $\begin{array}{l}\text { Haustechnik, } \\
\text { Solarthermie, } \\
\text { BHKW, } \\
\text { Wärmepumpen }\end{array}$ & Systemvertrieb \\
\hline ESP GmbH & $\begin{array}{l}\text { Erneuerbare Wärme } \\
\text { \& Strom, Installation }\end{array}$ & $\begin{array}{l}\mathrm{HH}, \mathrm{GHD}, \text { ffentl. } \\
\text { Unternehmen }\end{array}$ & $\begin{array}{l}\text { Servicegeb hren, } \\
\text { Asset-Verkauf }\end{array}$ & $\begin{array}{l}\text { Wohnen, } \\
\text { Service }\end{array}$ & $\begin{array}{l}\text { Technologieanbieter, } \\
\text { Anlagenbetreiber, } \\
\text { lokales Handwerk }\end{array}$ & $\begin{array}{l}\text { Klein-Solarthermie, } \\
\text { Photovoltaik-Klein }\end{array}$ & Systemvertrieb \\
\hline $\begin{array}{l}\text { Getriebebau } \\
\text { NORD GmbH \& } \\
\text { Co. KG }\end{array}$ & Anlagenkomponenten & $\begin{array}{l}\text { Anlagenbauer, } \\
\text { Industrie, EVU, } \\
\text { Versorger, GHD }\end{array}$ & Asset-Verkauf & $\begin{array}{l}\text { Technologie- } \\
\text { anbieter }\end{array}$ & $\begin{array}{l}\text { Technologieanbieter, } \\
\text { Anlagenbetreiber, EVU, } \\
\text { Projektierer }\end{array}$ & $\begin{array}{l}\text { Mess- und } \\
\text { Steuerungstechnik }\end{array}$ & $\begin{array}{l}\text { Systemtechnik- } \\
\text { produzent }\end{array}$ \\
\hline
\end{tabular}




\begin{tabular}{|c|c|c|c|c|c|c|c|}
\hline $\begin{array}{l}\text { Unselbstst ndige } \\
\text { Tankstelle } \\
\text { (ARAL) }\end{array}$ & $\begin{array}{l}\text { Kraftstoff- } \\
\text { bereitstellung, } \\
\text { Mobilität }\end{array}$ & Autonutzer & Handelsgewinne & $\begin{array}{l}\text { Vertrieb, } \\
\text { Handel, } \\
\text { Mobilität }\end{array}$ & $\begin{array}{l}\text { Messdienstleister, Erzeuger, } \\
\text { Abrechnungsdienstleister, } \\
\text { Händler, Datenverarbeiter }\end{array}$ & $\begin{array}{l}\text { Mess- und } \\
\text { Steuerungstechnik }\end{array}$ & $\begin{array}{l}\text { Endkunden- } \\
\text { vertrieb Energie }\end{array}$ \\
\hline $\begin{array}{l}\text { Unselbstst ndige } \\
\text { Tankstelle } \\
\text { (HEM) }\end{array}$ & $\begin{array}{l}\text { Kraftstoff- } \\
\text { bereitstellung, } \\
\text { Mobilität }\end{array}$ & Autonutzer & Handelsgewinne & $\begin{array}{l}\text { Vertrieb, } \\
\text { Handel, } \\
\text { Mobilität }\end{array}$ & $\begin{array}{l}\text { Messdienstleister, Erzeuger, } \\
\text { Abrechnungsdienstleister, } \\
\text { Händler, Datenverarbeiter }\end{array}$ & $\begin{array}{l}\text { Mess- und } \\
\text { Steuerungstechnik }\end{array}$ & $\begin{array}{l}\text { Endkunden- } \\
\text { vertrieb Energie }\end{array}$ \\
\hline $\begin{array}{l}\text { Netzdienststelle } \\
\text { der WEMAG }\end{array}$ & $\begin{array}{l}\text { Energietransport, } \\
\text { Marktzugang, } \\
\text { Versorgungs- } \\
\text { sicherheit, System- } \\
\text { dienstleistungen }\end{array}$ & $\begin{array}{l}\text { Erzeuger, } \\
\text { Verbraucher }\end{array}$ & $\begin{array}{l}\text { Netzentgelte, } \\
\text { Anschluss- } \\
\text { geb hren }\end{array}$ & $\begin{array}{l}\text { Verteilung, } \\
\text { Kapazit ts- } \\
\text { management }\end{array}$ & $\begin{array}{l}\text { Technologieanbieter, } \\
\text { Erzeuger, Verbraucher, } \\
\text { Netzbetreiber, Messstellen- } \\
\text { betreiber, Behörden, EVU, } \\
\text { Regulierer, Messdienstleister }\end{array}$ & $\begin{array}{l}\text { Netzinfrastruktur, } \\
\text { Mess- und } \\
\text { Steuerungstechnik }\end{array}$ & $\begin{array}{l}\text { Netzbetrieb } \\
\text { Grundmodell }\end{array}$ \\
\hline $\begin{array}{l}\text { Daniel Lorenz - } \\
\text { Heizung - Sa- } \\
\text { nit } r \text { - Solar }\end{array}$ & $\begin{array}{l}\text { (Erneuerbare) Wärme, } \\
\text { Komplettlösung - } \\
\text { Planung, Installation, } \\
\text { Wartung }\end{array}$ & $\begin{array}{l}\text { Haushalte, GHD, } \\
\text { Öffentl. Unternehmen }\end{array}$ & $\begin{array}{l}\text { Servicegebühren, } \\
\text { Asset-Verkauf }\end{array}$ & $\begin{array}{l}\text { Wohnen, } \\
\text { Service }\end{array}$ & $\begin{array}{l}\text { Technologieanbieter, } \\
\text { Anlagenbetreiber, } \\
\text { lokales Handwerk }\end{array}$ & $\begin{array}{l}\text { Biomasseanlagen, } \\
\text { Heiztechnik }\end{array}$ & Systemvertrieb \\
\hline Stromtankstelle & $\begin{array}{l}\text { Strom, Mobilität, } \\
\text { Anlagenbetrieb, } \\
\text { Marktzugang, } \\
\text { Abrechnung }\end{array}$ & Autonutzer & $\begin{array}{l}\text { Nutzungs- } \\
\text { gebühren, } \\
\text { Servicegebühren, } \\
\text { Provision, } \\
\text { Asset-Verkauf }\end{array}$ & $\begin{array}{l}\text { Vertrieb, } \\
\text { Mobilität, } \\
\text { Service }\end{array}$ & $\begin{array}{l}\text { Technologieanbieter, } \\
\text { Messdienstleister, } \\
\text { Softwareanbieter, } \\
\text { Automobilhersteller, } \\
\text { Datenplattform }\end{array}$ & $\begin{array}{l}\text { Ladeinfrastruktur, } \\
\text { Software, Mess- } \\
\text { und Steuerungs- } \\
\text { technik }\end{array}$ & $\begin{array}{l}\text { Power-to- } \\
\text { Mobility-Lade- } \\
\text { infrastruktur }\end{array}$ \\
\hline $\begin{array}{l}\text { HanseGas } \\
\text { GmbH }\end{array}$ & $\begin{array}{l}\text { Energietransport, } \\
\text { Marktzugang, } \\
\text { Versorgungs- } \\
\text { sicherheit, System- } \\
\text { dienstleistungen }\end{array}$ & $\begin{array}{l}\text { Erzeuger, } \\
\text { Verbraucher }\end{array}$ & $\begin{array}{l}\text { Netzentgelte, } \\
\text { Anschluss- } \\
\text { geb hren }\end{array}$ & $\begin{array}{l}\text { Verteilung, } \\
\text { Kapazit ts- } \\
\text { management }\end{array}$ & $\begin{array}{l}\text { Technologieanbieter, Erzeu- } \\
\text { ger, Verbraucher, Netzbetrei- } \\
\text { ber, Messstellenbetreiber, } \\
\text { Behörden, EVU, Regulierer, } \\
\text { Messdienstleister }\end{array}$ & $\begin{array}{l}\text { Netzinfrastruktur, } \\
\text { Mess- und } \\
\text { Steuerungstechnik }\end{array}$ & $\begin{array}{l}\text { Netzbetrieb } \\
\text { Grundmodell }\end{array}$ \\
\hline Werkers Welt & $\begin{array}{l}\text { Kraftstoff- } \\
\text { bereitstellung }\end{array}$ & $\begin{array}{l}\mathrm{HH}, \mathrm{GHD} \text {, Industrie, } \\
\text { Öffentl. Unternehmen }\end{array}$ & Handelsgewinne & $\begin{array}{l}\text { Vertrieb, } \\
\text { Handel }\end{array}$ & Erzeuger, Händler & keine & $\begin{array}{l}\text { Endkunden- } \\
\text { vertrieb Energie }\end{array}$ \\
\hline
\end{tabular}




\begin{tabular}{|c|c|c|c|c|c|c|c|}
\hline $\begin{array}{l}\text { Unselbstst ndige } \\
\text { Tankstelle } \\
\text { (Nord I L tzow) }\end{array}$ & $\begin{array}{l}\text { Kraftstoff- } \\
\text { bereitstellung, } \\
\text { Mobilität }\end{array}$ & Autonutzer & Handelsgewinne & $\begin{array}{l}\text { Vertrieb, } \\
\text { Handel, } \\
\text { Mobilität }\end{array}$ & $\begin{array}{l}\text { Messdienstleister, Erzeuger, } \\
\text { Abrechnungsdienstleister, } \\
\text { Händler, Datenverarbeiter }\end{array}$ & $\begin{array}{l}\text { Mess- und } \\
\text { Steuerungstechnik }\end{array}$ & $\begin{array}{l}\text { Endkunden- } \\
\text { vertrieb Energie }\end{array}$ \\
\hline $\begin{array}{l}\text { Block Heizung + } \\
\text { Sanitär GmbH } \\
\text { Gebr der Block }\end{array}$ & $\begin{array}{l}\text { (Erneuerbare) Wärme, } \\
\text { Komplettlösung - } \\
\text { Planung, Installation, } \\
\text { Wartung }\end{array}$ & $\begin{array}{l}\text { Haushalte, GHD, } \\
\text { Öffentl. Unternehmen }\end{array}$ & $\begin{array}{l}\text { Servicegebühren, } \\
\text { Asset-Verkauf }\end{array}$ & $\begin{array}{l}\text { Wohnen, } \\
\text { Service }\end{array}$ & $\begin{array}{l}\text { Technologieanbieter, } \\
\text { Anlagenbetreiber, } \\
\text { lokales Handwerk }\end{array}$ & $\begin{array}{l}\text { Biomasseanlagen, } \\
\text { Heiztechnik }\end{array}$ & Systemvertrieb \\
\hline $\begin{array}{l}\text { P.P.S. power } \\
\text { GmbH \& Co. } \\
\text { KG }\end{array}$ & $\begin{array}{l}\text { Anlagen- } \\
\text { komponenten }\end{array}$ & Industrie, EVU & $\begin{array}{l}\text { Asset-Verkauf, } \\
\text { Handelsgewinne }\end{array}$ & $\begin{array}{l}\text { Technologie- } \\
\text { anbieter }\end{array}$ & $\begin{array}{l}\text { Anlagenbetreiber, EVU, } \\
\text { Technologieanbieter }\end{array}$ & Nicht spezifiziert & $\begin{array}{l}\text { Technologie- } \\
\text { vertrieb }\end{array}$ \\
\hline S.E.N.Tech & $\begin{array}{l}\text { Erneuerbare Wärme, } \\
\text { Komplettlösung - } \\
\text { Beratung, Planung, } \\
\text { Installation, Wartung }\end{array}$ & $\begin{array}{l}\text { HH, GHD, } \\
\text { Öffentl. Unternehmen }\end{array}$ & $\begin{array}{l}\text { Servicegebühren, } \\
\text { Asset-Verkauf }\end{array}$ & $\begin{array}{l}\text { Wohnen, } \\
\text { Service }\end{array}$ & $\begin{array}{l}\text { Technologieanbieter, } \\
\text { Anlagenbetreiber, } \\
\text { lokales Handwerk }\end{array}$ & $\begin{array}{l}\text { Biomasseanlagen, } \\
\text { Wärmepumpen, } \\
\text { Solarthermie, } \\
\text { Heiztechnik }\end{array}$ & Systemvertrieb \\
\hline $\begin{array}{l}\text { Dabelstein Bio- } \\
\text { gasanlage GmbH } \\
\text { \& Co. KG }\end{array}$ & $\begin{array}{l}\text { Erneuerbare Wärme } \\
\text { \& Strom, } \\
\text { Klimaschutzbeitrag }\end{array}$ & $\begin{array}{l}\text { HH, GHD, Industrie, } \\
\text { Netzbetreiber, EVU, } \\
\text { H ndler, St dte und } \\
\text { Kommunen, ffentl. } \\
\text { Unternehmen }\end{array}$ & $\begin{array}{l}\text { Einspeise- } \\
\text { verg tung, } \\
\text { Grundgebühr, } \\
\text { Energiepreis }\end{array}$ & Erzeugung & $\begin{array}{l}\text { Netzbetreiber, Vermarkter, } \\
\text { Messstellenbetreiber, } \\
\text { Technologieanbieter, } \\
\text { EVU, Landwirtschaft }\end{array}$ & $\begin{array}{l}\text { Große erneuerbare } \\
\text { Erzeuger }\end{array}$ & $\begin{array}{l}\text { Erneuerbare } \\
\text { Erzeugung }\end{array}$ \\
\hline $\begin{array}{l}\text { Van der Ham \& } \\
\text { Co. KG }\end{array}$ & $\begin{array}{l}\text { Erneuerbare Wärme } \\
\text { \& Strom, } \\
\text { Klimaschutzbeitrag }\end{array}$ & $\begin{array}{l}\text { HH, GHD, Industrie, } \\
\text { Netzbetreiber, EVU, } \\
\text { H ndler, St dte und } \\
\text { Kommunen, ffentl. } \\
\text { Unternehmen }\end{array}$ & $\begin{array}{l}\text { Einspeise- } \\
\text { verg tung, } \\
\text { Grundgebühr, } \\
\text { Energiepreis }\end{array}$ & Erzeugung & $\begin{array}{l}\text { Netzbetreiber, Vermarkter, } \\
\text { Messstellenbetreiber, } \\
\text { Technologieanbieter, } \\
\text { EVU, Landwirtschaft }\end{array}$ & $\begin{array}{l}\text { Große erneuerbare } \\
\text { Erzeuger }\end{array}$ & $\begin{array}{l}\text { Erneuerbare } \\
\text { Erzeugung }\end{array}$ \\
\hline $\begin{array}{l}\text { VEB M ritz } \\
\text { Holz GmbH }\end{array}$ & $\begin{array}{l}\text { Erneuerbare Brenn- } \\
\text { stoffe, Regionale } \\
\text { Wertschöpfung }\end{array}$ & $\begin{array}{l}\text { HH, GHD, } \\
\text { Erzeuger, Händler }\end{array}$ & Energiepreis & $\begin{array}{l}\text { Erzeugung, } \\
\text { Vertrieb }\end{array}$ & $\begin{array}{l}\text { Händler, Technologie- } \\
\text { anbieter, Biomasseanbieter, } \\
\text { Landwirtschaft }\end{array}$ & Energieholzwerk & $\begin{array}{l}\text { Erneuerbare } \\
\text { Brennstoffe }\end{array}$ \\
\hline
\end{tabular}




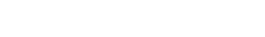

\begin{tabular}{|c|c|c|c|c|c|c|c|}
\hline $\begin{array}{l}\text { Buhl Tankstellen } \\
\text { \& Service GmbH }\end{array}$ & $\begin{array}{l}\text { Kraftstoff- } \\
\text { bereitstellung, } \\
\text { Mobilität }\end{array}$ & Autonutzer & Handelsgewinne & $\begin{array}{l}\text { Vertrieb, } \\
\text { Handel, } \\
\text { Mobilität }\end{array}$ & $\begin{array}{l}\text { Messdienstleister, Erzeuger, } \\
\text { Abrechnungsdienstleister, } \\
\text { Händler, Datenverarbeiter }\end{array}$ & $\begin{array}{l}\text { Mess- und } \\
\text { Steuerungstechnik }\end{array}$ & $\begin{array}{l}\text { Endkunden- } \\
\text { vertrieb Energie }\end{array}$ \\
\hline $\begin{array}{l}\text { Zweite Bioener- } \\
\text { gie B tow GmbH } \\
\text { \& Co. KG }\end{array}$ & $\begin{array}{l}\text { Erneuerbare Wärme } \\
\text { \& Strom, } \\
\text { Klimaschutzbeitrag }\end{array}$ & $\begin{array}{l}\mathrm{HH}, \mathrm{GHD} \text {, Industrie, } \\
\text { Netzbetreiber, EVU, } \\
\text { H ndler, St dte und } \\
\text { Kommunen, ffentl. } \\
\text { Unternehmen }\end{array}$ & $\begin{array}{l}\text { Einspeise- } \\
\text { verg tung, } \\
\text { Grundgebühr, } \\
\text { Energiepreis }\end{array}$ & Erzeugung & $\begin{array}{l}\text { Netzbetreiber, Vermarkter, } \\
\text { Messstellenbetreiber, } \\
\text { Technologieanbieter, } \\
\text { EVU, Landwirtschaft }\end{array}$ & $\begin{array}{l}\text { Große erneuerbare } \\
\text { Erzeuger }\end{array}$ & $\begin{array}{l}\text { Erneuerbare } \\
\text { Erzeugung }\end{array}$ \\
\hline $\begin{array}{l}\text { Elde-Wind } \\
\text { GmbH \& Co. } \\
\text { KG }\end{array}$ & $\begin{array}{l}\text { Erneuerbarer Strom, } \\
\text { Klimaschutzbeitrag }\end{array}$ & $\begin{array}{l}\text { HH, GHD, Industrie, } \\
\text { EVU, H ndler, St dte } \\
\text { und Kommunen, } \\
\text { ffentl. Unternehmen }\end{array}$ & $\begin{array}{l}\text { Einspeise- } \\
\text { verg tung, } \\
\text { Energiepreis }\end{array}$ & Erzeugung & $\begin{array}{l}\text { Netzbetreiber, Vermarkter, } \\
\text { Messstellenbetreiber, } \\
\text { Technologieanbieter, EVU }\end{array}$ & $\begin{array}{l}\text { Große erneuerbare } \\
\text { Erzeuger }\end{array}$ & $\begin{array}{l}\text { Erneuerbare } \\
\text { Erzeugung }\end{array}$ \\
\hline $\begin{array}{l}\text { Windfeld } \\
\text { B tow/Zepkow } \\
\text { Gmbh }\end{array}$ & $\begin{array}{l}\text { Erneuerbaren Strom, } \\
\text { Klimaschutzbeitrag }\end{array}$ & $\begin{array}{l}\text { HH, GHD, Industrie, } \\
\text { EVU, H ndler, St dte } \\
\text { und Kommunen, } \\
\text { ffentl. Unternehmen }\end{array}$ & $\begin{array}{l}\text { Einspeise- } \\
\text { verg tung, } \\
\text { Energiepreis }\end{array}$ & Erzeugung & $\begin{array}{l}\text { Technologieanbieter, } \\
\text { Netzbetreiber, } \\
\text { Messstellenbetreiber, } \\
\text { EVU, Vermarkter, } \\
\text { Projektierer }\end{array}$ & $\begin{array}{l}\text { Große erneuerbare } \\
\text { Erzeuger }\end{array}$ & $\begin{array}{l}\text { Erneuerbare } \\
\text { Erzeugung }\end{array}$ \\
\hline $\begin{array}{l}\text { Bj rn Wilken } \\
\text { GmbH }\end{array}$ & $\begin{array}{l}\text { Erneuerbare Wärme, } \\
\text { Komplettlösung - } \\
\text { Beratung, Planung, } \\
\text { Installation, Wartung }\end{array}$ & $\begin{array}{l}\text { Haushalte, GHD, } \\
\text { Öffentl. Unternehmen }\end{array}$ & $\begin{array}{l}\text { Servicegebühren, } \\
\text { Asset-Verkauf }\end{array}$ & $\begin{array}{l}\text { Wohnen, } \\
\text { Service }\end{array}$ & $\begin{array}{l}\text { Technologieanbieter, } \\
\text { Anlagenbetreiber, } \\
\text { lokales Handwerk }\end{array}$ & $\begin{array}{l}\text { Heiztechnik, } \\
\text { Solarthermie, } \\
\text { Wärmepumpen, } \\
\text { Geothermie, } \\
\text { Biomasseanlagen }\end{array}$ & Systemvertrieb \\
\hline $\begin{array}{l}\text { Hochst dt \& } \\
\text { Behncke Service } \\
\text { GmbH }\end{array}$ & $\begin{array}{l}\text { Erneuerbare Wärme, } \\
\text { Komplettlösung - } \\
\text { Beratung, Planung, } \\
\text { Installation, Wartung }\end{array}$ & $\begin{array}{l}\text { Haushalte, GHD, } \\
\text { Öffentl. Unternehmen }\end{array}$ & $\begin{array}{l}\text { Servicegebühren, } \\
\text { Asset-Verkauf }\end{array}$ & $\begin{array}{l}\text { Wohnen, } \\
\text { Service }\end{array}$ & $\begin{array}{l}\text { Technologieanbieter, } \\
\text { Anlagenbetreiber, } \\
\text { lokales Handwerk }\end{array}$ & $\begin{array}{l}\text { Heiztechnik, Solar- } \\
\text { thermie, Wärme- } \\
\text { pumpen, } \\
\text { Biomasseanlagen }\end{array}$ & Systemvertrieb \\
\hline
\end{tabular}




\begin{tabular}{|c|c|c|c|c|c|c|c|}
\hline $\begin{array}{l}\text { KWE New } \\
\text { Energy GmbH }\end{array}$ & $\begin{array}{l}\text { Erneuerbaren Strom, } \\
\text { Klimaschutzbeitrag }\end{array}$ & $\begin{array}{l}\text { HH, GHD, Industrie, } \\
\text { EVU, H ndler, St dte } \\
\text { und Kommunen, } \\
\text { ffentl. Unternehmen }\end{array}$ & $\begin{array}{l}\text { Einspeise- } \\
\text { verg tung, } \\
\text { Energiepreis }\end{array}$ & Erzeugung & $\begin{array}{l}\text { Technologieanbieter, EVU, } \\
\text { Messstellenbetreiber, } \\
\text { Vermarkter, Netzbetreiber, } \\
\text { Projektierer }\end{array}$ & $\begin{array}{l}\text { Große erneuerbare } \\
\text { Erzeuger }\end{array}$ & $\begin{array}{l}\text { Erneuerbare } \\
\text { Erzeugung }\end{array}$ \\
\hline $\begin{array}{l}\text { WSG Energy } \\
\text { GmbH }\end{array}$ & $\begin{array}{l}\text { Komplettlösung - } \\
\text { Erneuerbare } \\
\text { Erzeugungsanlagen }\end{array}$ & $\begin{array}{l}\text { Erzeuger, Energie- } \\
\text { genossenschaften, } \\
\text { Öffentl. } \\
\text { Unternehmen, EVU }\end{array}$ & $\begin{array}{l}\text { Servicegebühren, } \\
\text { Asset-Verkauf }\end{array}$ & Service & $\begin{array}{l}\text { Technolologievertriebe, } \\
\text { Behörden, Netzbetreiber, } \\
\text { Finanzierer, Baugewerbe }\end{array}$ & $\begin{array}{l}\text { Große erneuerbare } \\
\text { Erzeuger }\end{array}$ & $\begin{array}{l}\text { Erneuerbare } \\
\text { Projektierung }\end{array}$ \\
\hline $\begin{array}{l}\text { Buhl Tankstellen } \\
\text { \& Service GmbH }\end{array}$ & $\begin{array}{l}\text { Kraftstoff- } \\
\text { bereitstellung, } \\
\text { Mobilität }\end{array}$ & Autonutzer & Handelsgewinne & $\begin{array}{l}\text { Vertrieb, } \\
\text { Handel, } \\
\text { Mobilität }\end{array}$ & $\begin{array}{l}\text { Messdienstleister, Erzeuger, } \\
\text { Abrechnungsdienstleister, } \\
\text { Händler, Datenverarbeiter }\end{array}$ & $\begin{array}{l}\text { Mess- und } \\
\text { Steuerungstechnik }\end{array}$ & $\begin{array}{l}\text { Endkunden- } \\
\text { vertrieb Energie }\end{array}$ \\
\hline $\begin{array}{l}\text { E.ON Edis Regi- } \\
\text { onalbereich } \\
\text { Mecklenburgi- } \\
\text { sche Seenplatte }\end{array}$ & $\begin{array}{l}\text { Datenauswertung, } \\
\text { Abrechnung, Kosten- } \\
\text { zuordnung, } \\
\text { Verbrauchsdaten, } \\
\text { Tarifmanagement }\end{array}$ & EVU & Servicegebühren & Service & $\begin{array}{l}\text { Softwareanbieter, } \\
\text { Messstellenbetreiber, } \\
\text { Messdienstleister }\end{array}$ & $\begin{array}{l}\text { Software, } \\
\text { Datenplattform }\end{array}$ & $\begin{array}{l}\text { Abrechnungs- } \\
\text { service }\end{array}$ \\
\hline $\begin{array}{l}\text { Technik Center } \\
\text { R bel Gmbh \& } \\
\text { Co. KG }\end{array}$ & $\begin{array}{l}\text { Kraftstoff- } \\
\text { bereitstellung, } \\
\text { Mobilität }\end{array}$ & Autonutzer & Handelsgewinne & $\begin{array}{l}\text { Vertrieb, } \\
\text { Handel, } \\
\text { Mobilität }\end{array}$ & $\begin{array}{l}\text { Messdienstleister, } \\
\text { Abrechnungsdienstleister, } \\
\text { Händler, Datenverarbeiter }\end{array}$ & $\begin{array}{l}\text { Mess- und } \\
\text { Steuerungstechnik }\end{array}$ & $\begin{array}{l}\text { Endkunden- } \\
\text { vertrieb Energie }\end{array}$ \\
\hline $\begin{array}{l}\text { Montagefirma } \\
\text { Frank Wilke }\end{array}$ & $\begin{array}{l}\text { Erneuerbaren Strom } \\
\& \text { Wärme }\end{array}$ & $\begin{array}{l}\text { Haushalte, GHD, } \\
\text { Öffentl. Unternehmen }\end{array}$ & $\begin{array}{l}\text { Servicegebühren, } \\
\text { Asset-Verkauf }\end{array}$ & $\begin{array}{l}\text { Wohnen, } \\
\text { Service }\end{array}$ & $\begin{array}{l}\text { Technologieanbieter, } \\
\text { Anlagenbetreiber, } \\
\text { lokales Handwerk }\end{array}$ & $\begin{array}{l}\text { Photovoltaik-Klein, } \\
\text { Solarthermie }\end{array}$ & Systemvertrieb \\
\hline TimberMacPom & $\begin{array}{l}\text { Erneuerbare Brenn- } \\
\text { stoffe, Regionale } \\
\text { Wertschöpfung }\end{array}$ & $\begin{array}{l}\text { HH, GHD, } \\
\text { Erzeuger, Händler }\end{array}$ & Energiepreis & $\begin{array}{l}\text { Erzeugung, } \\
\text { Vertrieb }\end{array}$ & $\begin{array}{l}\text { Händler, Technologie- } \\
\text { anbieter, Biomasseanbieter, } \\
\text { Landwirtschaft }\end{array}$ & Energieholzwerk & $\begin{array}{l}\text { Erneuerbare } \\
\text { Brennstoffe }\end{array}$ \\
\hline
\end{tabular}


Haustechnik

Erneuerbare Wärme

Haushalte, GHD,

Servicegebühren,

Wohnen,

Technologieanbieter,

Solarthermie,

Systemvertrieb

\section{Nessau}

\& Strom, Komplettlö-

Öffentl. Unternehmen

Asset-Verkauf

Service

Anlagenbetreiber,

lokales Handwerk

Photovoltaik-Klein,

$$
\text { sung }
$$

Energieberatung,

HH, GHD, Industrie,

Servicegeb hren

Service,

Technologieanbieter,

Behörden, Finanzierer,

Wärmepumpen,

Biomasseanlagen,

Heiztechnik,

Haustechnik

st tzpunkt

Kostenoptimierung,

Öffentl.

Verbrauch

Datenverarbeiter,

Software, Mess-

Energieberatung

\section{$\mathrm{R}$ bel/M ritz}

Maßnahmen-

Unternehmen, Städte

Messdienstleister

und Steuerungs-

Erweiterung

\section{der Verbrau-}

identifikation,

und Kommunen,

technik

cherzentrale

Effizienzsteigerung

Wohnungsbau-

gesellschaften

\section{Legende}

Veränderte Ausprägung (aus Pool)

Neue Ausprägung (nicht aus Pool) 
8.2 Anhang 2: Auflistung der Gemeindenamen

Tabelle 2: Liste der Untersuchungsgemeinden

\begin{tabular}{l|c|c|l|l}
\multicolumn{1}{|c|}{ Gemeindename } & $\begin{array}{c}\text { Postleitzahl der Ge- } \\
\text { meindeverwaltung }\end{array}$ & Gemeindeschlüssel & Verwaltungsgemeinschaft & \multicolumn{1}{|c}{ Landkreis } \\
\hline Rehna, Stadt & 19217 & 13074065 & Amt Rehna & Nordwestmecklenburg \\
Gadebusch, Stadt & 19205 & 13074021 & Amt Gadebusch & Nordwestmecklenburg \\
Lützow & 19209 & 13074050 & Amt Lützow-Lübstorf & Nordwestmecklenburg \\
Lübsdorf & 19069 & 13074048 & Amt Lützow-Lübstorf & Nordwestmecklenburg \\
\hline Alt Meteln & 19069 & 13074001 & Amt Lützow-Lübstorf & Nordwestmecklenburg \\
\hline Klein Trebbow & 19069 & 13074038 & Amt Lützow-Lübstorf & Nordwestmecklenburg \\
\hline Brüsewitz & 19071 & 13074012 & Amt Lützow-Lübstorf & Nordwestmecklenburg \\
\hline Bollewick & 17207 & 13071013 & Amt Röbel-Müritz & Mecklenburgische Seenplatte \\
Fincken & 17209 & 13071034 & Amt Röbel-Müritz & Mecklenburgische Seenplatte \\
\hline Wredenhagen & 17209 & 13071165 & Amt Röbel-Müritz & Mecklenburgische Seenplatte \\
\hline Leizen & 17209 & Amt Röbel-Müritz & Mecklenburgische Seenplatte \\
\hline Buchholz & 17209 & 13071020 & Amt Röbel-Müritz & Mecklenburgische Seenplatte \\
\hline Grabow-Below & 17209 & 13071046 & Amt Röbel-Müritz & Mecklenburgische Seenplatte \\
\hline Melz & 17209 & 13071168 & Amt Röbel-Müritz & Mecklenburgische Seenplatte \\
\hline Zepkow & 17209 & 13071023 & Amt Röbel-Müritz & Mecklenburgische Seenplatte \\
Bütow & 17209 & 13071073 & Amt Röbel-Müritz & Mecklenburgische Seenplatte \\
\hline Kieve & 17209 & 13071091 & Amt Röbel-Müritz & Mecklenburgische Seenplatte \\
\hline Ludorf & 17207 & 13071124 & Amt Röbel-Müritz & Mecklenburgische Seenplatte \\
\hline Röbel/Müritz, Stadt & 17207 & &
\end{tabular}

Quelle: Eigene Darstellung mit Daten des statistischen Bundesamts (2018) 